\title{
Dual-phase steel sheets under cyclic tension-compression to large strains: Experiments and crystal plasticity modeling
}

\author{
Milovan Zecevic ${ }^{\mathrm{a}}$, Yannis P. Korkolis ${ }^{\mathrm{a}}$, Toshihiko Kuwabara ${ }^{\mathrm{b}}$, Marko Knezevic ${ }^{\mathrm{a},{ }^{*}}$ \\ a'Department of Mechanical Engineering, University of New Hampshire, Durham, NH 03824, \\ USA \\ ${ }^{\mathrm{b}}$ Division of Advanced Mechanical Systems Engineering, Institute of Engineering, Tokyo \\ University of Agriculture and Technology, Tokyo 184-8588, Japan
}

\begin{abstract}
In this work, we develop a physically-based crystal plasticity model for the prediction of cyclic tension-compression deformation of multi-phase materials, specifically dual-phase (DP) steels. The model is elasto-plastic in nature and integrates a hardening law based on statistically stored dislocation density, localized hardening due to geometrically necessary dislocations (GNDs), slip-system-level kinematic backstresses, and annihilation of dislocations. The model further features a two level homogenization scheme where the first level is the overall response of a two-phase polycrystalline aggregate and the second level is the homogenized response of the martensite polycrystalline regions. The model is applied to simulate a cyclic tensioncompression-tension deformation behavior of DP 590 steel sheets. From experiments, we observe that the material exhibits a typical decreasing hardening rate during forward loading, followed by a linear and then a non-linear unloading upon the load reversal, the Bauschinger effect, and changes in hardening rate during strain reversals. To predict these effects, we identify the model parameters using a portion of the measured data and validate and verify them using the remaining data. The developed model is capable of predicting all the particular features of the cyclic deformation of DP 590 steel, with great accuracy. From the predictions, we infer and discuss the effects of GNDs, the backstresses, dislocation annihilation, and the two-level homogenization scheme on capturing the cyclic deformation behavior of the material.
\end{abstract}

Keywords: A. Microstructures; A. Dislocations; B. Constitutive behavior; B. Cyclic loading; Steel DP590

\footnotetext{
* Corresponding author at: University of New Hampshire, Department of Mechanical Engineering, 33 Academic Way, Kingsbury Hall, W119, Durham, New Hampshire 03824, United States. Tel.: 603862 5179; fax: 603862 1865.

E-mail address: marko.knezevic@unh.edu (M. Knezevic).
} 


\section{Introduction}

In order to arrive at a solution of the governing equations of continuum mechanics such as the balance of linear momentum, an appropriate constitutive law describing the material behavior from its internal constitution under the action of applied deformation is required. The solution in terms of stress and strain measures is usually obtained numerically using full-field methods such as finite element (FE) methods (Bathe, 1996) or mean-field methods such as self-consistent (SC) models (Eshelby, 1957). The accuracy of the numerical solution is strongly dependent on the accuracy of constitutive law selected, also called a material model. Complex multiaxial deformation paths exerted on the material are routinely encountered in simulations of metal forming processes (Hosford and Caddell, 1993) putting stringent demands on the sensitivity of material models to strain path changes and the underlying path dependence of plastic deformation (Li et al., 2002).

Plastic deformation of polycrystalline metals induces anisotropy in the mechanical response, which oftentimes manifests itself in strong changes of the response characteristics upon change in the loading direction. Reversal of the strain path is achieved by applying strain in the opposite direction to that applied during forward pre-straining. Upon application of strain in the reversed direction, the material exhibits non-linear unloading (Cullen and Korkolis, 2013; Deng et al., 2015; Wagoner et al., 2013; Yoshida et al., 2002) and reduction of yield stress from the one reached at the end of pre-straining followed by rapid hardening known as the Bauschinger effect (BE) (Bauschinger, 1886). Additionally, the hardening rate that follows with continuation of straining in the reverse direction is also different from that during monotonic pre-straining. This stress offset between forward and reversed flow is referred to as the permanent softening (Hasegawa et al., 1975; Zang et al., 2013). These characteristics of material behavior are governed by the evolution of the underlying physical phenomena within the material microstructure, which are briefly summarized below. In particular, the presence of a second phase in the microstructure is known to enhance the non-linear unloading, the $\mathrm{BE}$ and the permanent softening effects (Bate and Wilson, 1986; Beyerlein, 2008).

First, the unloading of a pre-strained material consists of an initial linear elastic response, followed by a small nonlinear departure from the linear response (Deng et al., 2015; Pavlina et al., 2015; Sritharan and Chandel, 1997; Yoshida et al., 2002). The origin of the nonlinear unloading is in the partial re-emission of dislocations impeded by grain and phase boundaries during forward loading (Mompiou et al., 2012; Sritharan and Chandel, 1997). Dislocations incorporated in the boundaries during forward loading are referred to as the dislocation pile-ups. The re-emission process of the loosely-tangled dislocation population from the pile-ups is facilitated by the relaxation of micro backstresses arising from the dislocations pile-ups during unloading (Sritharan and Chandel, 1997). The level of pre-strain achieved during forward loading increases the magnitude of deviation from the linear elastic unloading behavior. Hence, to predict the non-linear unloading, material models must first accurately model pre-straining.

Second, the BE is observed and extensively studied in both single crystal (Demir and Raabe, 2010; Gough et al., 1927) and polycrystalline metals (Abel, 1987; Nieh and Nix, 1986; Stout and 
Rollett, 1990; Verma et al., 2011). The origin of BE in single crystals is a backstress having intra-granular source. This backstress arises from the incompatibility between hard regions of dislocation cell walls and soft regions of cell interiors (Kassner et al., 2013; Mughrabi, 1983). These long range internal stresses are known as the type III stresses. The built up of the type III stresses (Withers and Bhadeshia, 2001) acts against the applied stress during forward loading. Upon loading in the reverse direction, the applied stresses combine with the backstresses, which results in a drop of the reverse yield stress. Orowan's theory states that there is an anisotropy in resistance to dislocation motion between forward and reverse motion, and thus, it offers an additional explanation of the BE in single crystals (Orowan, 1959). Intuitively, dislocations move easier in the reversed direction because obstacles on the same path have been overcome during the forward motion. The mechanism causing the BE in single crystals is partly responsible for the $\mathrm{BE}$ in polycrystalline materials.

In single-phase polycrystalline metals backstresses, develop from interactions between individual grains of different crystal orientation during deformation. The origin of these backstresses is the anisotropy of grains with different crystal orientation, which causes varying properties over a material volume. These intergranular stresses are known as the type II stresses. A harder grain surrounded by softer grains will undergo lower plastic deformation than its surrounding neighbors. Incompatibility of accommodated plastic strain between the grains causes accumulation of dislocations around the strong grain, which results in the plastic strain gradient. The dislocations creating the gradient are referred to as the geometrically necessary dislocations (GNDs) (Bayley et al., 2006; Fleck et al., 1994).

The type II intergranular stresses act concurrently with intra-granular backstresses at phase boundaries in a multi-phase material. However, the density of GNDs surrounding grains of a harder phase inside grains of a softer phase is much larger than in single-phase grains due to typically large strength incompatibilities between grains of different phases than grains of the same phase and, thus, have a strong influence on the local and overall mechanical behavior of the multi-phase material (Brown and Stobbs, 1971; Kadkhodapour et al., 2011; Nesterova et al., 2015; Taupin et al., 2013). The GNDs layer of increased dislocation density induces directional internal stress originating from the layer to surrounding regions, which combines with the intergranular backstresses in individual phases, intra-granular backstresses in single crystals and the applied loading to determine the overall material behavior.

Third, the permanent softening effect originates from the annihilation/dissolution of the loosely-tangled dislocations contained in dislocation substructures such as cell walls formed during the primary deformation path, as well as the slow buildup of new dislocation substructures during deformation in the opposite direction (Kitayama et al., 2013; Stout and Rollett, 1990; Wilson et al., 1990). The presence of a harder second phase enhances the process of dislocation annihilation during reverse loading (Bate and Wilson, 1986; Gardey et al., 2005; Hasegawa et al., 1975; Wilson and Bate, 1986).

Simple phenomenological material models that are based on isotropic continuum plasticity are recognized to be incapable of capturing the above summarized effects (Armstrong and 
Frederick, 1966; Li et al., 2002), which motivated the development of more sophisticated phenomenological models. A range of models combining isotropic and linear or non-linear kinematic hardening laws have been developed (Armstrong and Frederick, 1966; Chaboche and Rousselier, 1983; Chaboche, 1977; Chaboche, 2008; Hu et al., 1992; McDowell, 1992). These phenomenological models, while computationally efficient and relatively easy to implement within FE codes, are not physically-based and therefore do not directly account for the mechanistic sources of backstresses and dislocation processes. A significant limitation of these models is that it is difficult to identify the values of model parameters, which demands expensive and complex mechanical tests and inverse methodologies to fit the parameters (Smith et al., 2014). Additionally, these models apply only to a specific material state and, sometimes, to the specific loading conditions used in the model fitting process.

Physics-based crystal plasticity constitutive models have been extensively used in understanding and predicting the deformation behavior of polycrystalline metals. These models are based on the crystallography of various deformation mechanisms and consider crystallographic orientation of individual grains. Polycrystal plasticity models link the response of the constituent single crystals to the response of a polycrystalline aggregate. To this end, different homogenization schemes exist ranging from an upper-bound Taylor (Al-Harbi et al., 2010; Knezevic et al., 2009; Knezevic et al., 2008; Knezevic and Savage, 2014; Savage and Knezevic, 2015; Taylor, 1938), to lower-bound Sachs (Fast et al., 2008; Knezevic and Kalidindi, 2007; Sachs, 1929), to mean-field self-consistent (SC) (Lebensohn and Tomé, 1993), and finally to full-field FE models (Kalidindi et al., 1992; Kalidindi et al., 2006; Knezevic et al., 2014c; Knezevic et al., 2013c; Knezevic et al., 2010; Roters et al., 2010; Zecevic et al., 2016a, b). To facilitate simulations under heterogeneous plastic strains, these polycrystal codes are further coupled with commercial finite element codes to operate at a FE integration point (Jahedi et al., 2015a; Jahedi et al., 2015b; Jahedi et al., 2014; Knezevic et al., 2014d; Knezevic et al., 2013d; Zecevic et al., 2015b, c). Polycrystal plasticity models intrinsically account for the anisotropic elasto-plastic behavior of the material due to preferred texture formation and are capable of calculating the inter-granular sources of backstress (type II). Moreover, these models are not constrained to an initial material state or to a specific deformation path, and therefore are much more predictive and robust than macroscopic plasticity models. Specifically, SC models have been successfully used to model strain path changes of single-phase materials and have proven capable of predicting the directional effects in mechanical response (Beyerlein, 2008; Beyerlein and Tomé, 2007; Kitayama et al., 2013; Knezevic et al., 2013a; Wen et al., 2014; Wollmershauser et al., 2012; Zecevic et al., 2015a). In recent work, we have developed a crystallography-based model within the elasto-plastic SC (EPSC) homogenization, integrating a dislocation-based hardening model and accounting for inter-granular stresses and slip system level backstresses, which made it capable of capturing non-linear unloading and the BE (Zecevic and Knezevic, 2015). The model also featured dissolution of the dislocation population upon load reversal, which enabled it to predict the change in hardening rate during reverse loading from that during forward loading. 
In present work, we extend the above mentioned single-phase EPSC model for cyclic deformation to treat particul features of the cyclic deformation of two-phase materials. While SC models have been used in modeling two-phase materials (Cai et al., 2009; Schwindt et al., 2015; Taupin et al., 2013; Yoshida et al., 2011), they lack rigorous treatment of phase-to-phase interaction effects arising during strain path changes. Specifically, the new two-phase EPSC model incorporates the directional effects at the slip system level through the slip system backstress kinematic hardening in every phase, GND layer around a harder phase, and two-level SC homogenization in order to better capture the effect of harder phase on the overall cyclic material behavior. The model retains the characteristic of the recently developed model for single-phase materials (Zecevic and Knezevic, 2015) in terms of accounting for the intergranular stresses of type II, evolving the slip system resistances based on the evolution of explicit dislocation density, and the consideration of dislocation dissolution related microstructural rearrangements upon load reversal.

To demonstrate the predictive capabilities of the model, we have measured the cyclic tension-compression-tension response to large strains of dual-phase steel (DP590) sheets and used the dataset for calibration and verification. The developed model successfully predicted the hardening during forward tension followed by non-linear unloading, the BE, and the subsequent hardening during the first reloading in the opposite direction, as well as the same during the second reloading in the original direction. Furthermore, the model predicted the cyclic material behavior not only as a function of the loading directions, but also as a function of the level of pre-strain. The model revealed that: (1) the backstresses control the non-linear unloading and the $\mathrm{BE}$, (2) the annihilation of dislocations plays the major role in capturing the BE and the shifts in the hardening rates during reverse loading, and (3) consideration of the plastic deformation of the martensite phase aided by the GND layer was essential for accurate prediction of stress levels in that phase, as well as the overall material response of DP 590 steel.

The paper is organized as follows. The cyclic tension-compression testing procedure, the measured data and the characterization data of the initial microstructure of the DP590 steel sheets are presented in section 2. The model incorporating the new physics of cyclic material behavior is described in section 3. Results of the cyclic simulations are presented in section 4 . The role of the individual physical features incorporated in the model reveled through the comparisons between simulated and measured cyclic curves is presented in section 5. Conclusions are presented in section 6.

\section{Material and experiments}

The material investigated in this study is a dual-phase steel. Dual-phase steels offer an outstanding combination of strength and formability as a result of their microstructure that consists of a hard martensitic phase dispersed in a soft ferritic matrix. The former phase provides the strengthening element within the latter phase. The latter phase ensures good cold formability. The particular dual-phase steel studied here is the DP590 steel manufactured and supplied by the 
United States Steel Corporation. The material was supplied in the form of galvannealed sheets of $2 \mathrm{~mm}$ thickness.

\subsection{Material characterization}

Electron backscatter diffraction (EBSD) orientation imaging was used to acquire the texture of the unstrained material, determine the volume fraction and morphology of martensite. The material was mechanically prepared for EBSD using automated sanding and polishing procedures. For sanding 240, 320, 400, 600, 800, and $1200 \mathrm{SiC}$ papers were used. After sanding the samples were polished using a sequence of oil-based diamond suspensions of 6,3 , and $1 \mu \mathrm{m}$, respectively. Fine-polishing of the samples was achieved with $0.06 \mu \mathrm{m}$ colloidal silica suspension. The automated EBSD data collection was performed using the Pegasus system (Octane Plus SDD detector and Hikari High Speed Camera) attached to a Tescan Lyra (Ga) field emission scanning electron microscope (SEM) at a voltage of $20 \mathrm{kV}$. The EBSD scans were run with $0.1 \mu \mathrm{m}$ and $0.3 \mu \mathrm{m}$ step size for small and large scans, respectively.

Figure 1a shows a typical inverse pole figure (IPF) map of DP590 steel. Additionally, several IPF maps of martensite regions are inserted to depict the hierarchical polycrystalline grain structure in these regions. We observe that occasionally martensite appears as a single grain and more often as a polycrystalline region consisting of several orientation variants related to their parent austenite grain. Figure 1b shows the corresponding image quality (IQ) map. Regions of martensite have lower IQ and are seen as dark regions in the IQ map, as noted earlier in (Pinard et al., 2013). The volume fraction of martensite is evaluated to be approximately $7.7 \%$ using a threshold procedure to distinguish between ferrite and martensite phase from the IQ map. The EBSD scans used to obtain representative texture information were run over a much larger area. The pole figures showing texture are presented in Fig. 1c. They confirm that the sheet exhibits a typical rolling texture of moderate intensity. 
$\mathrm{a}$

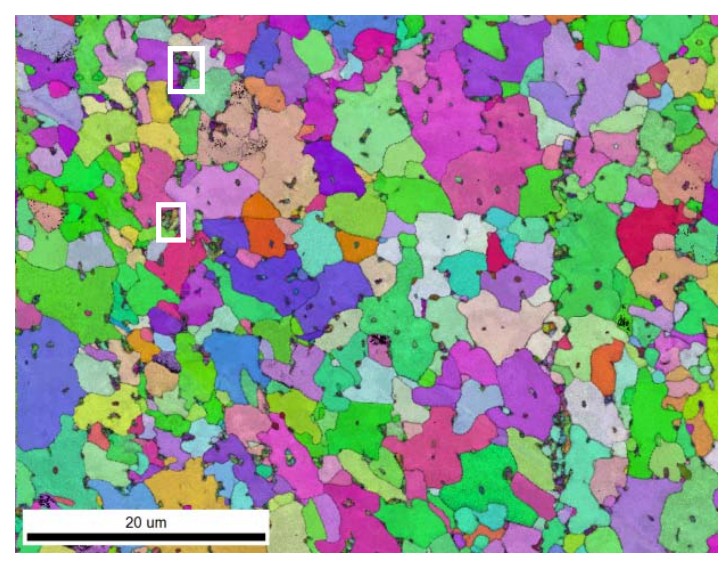

b

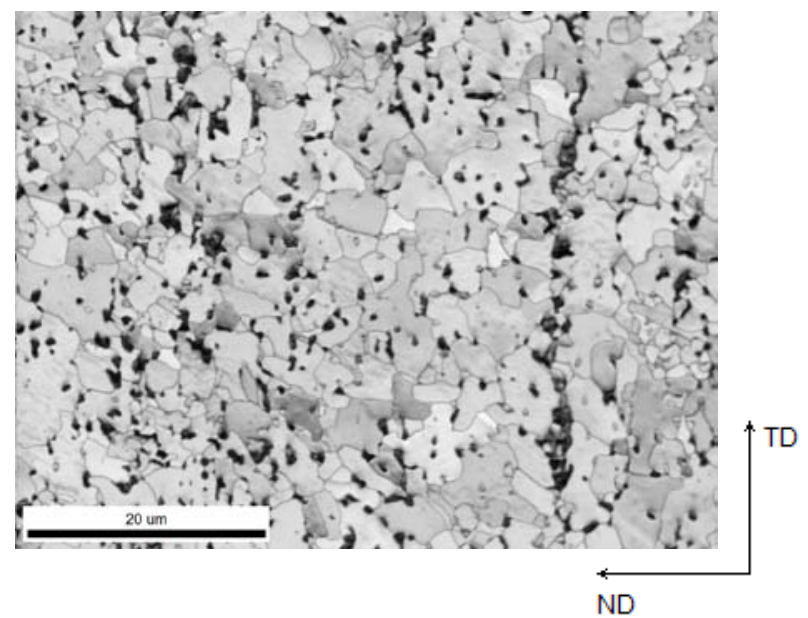

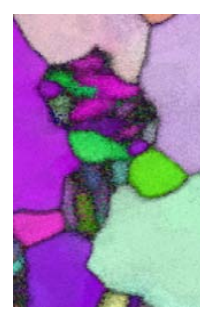

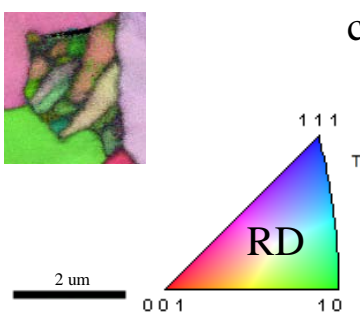

C
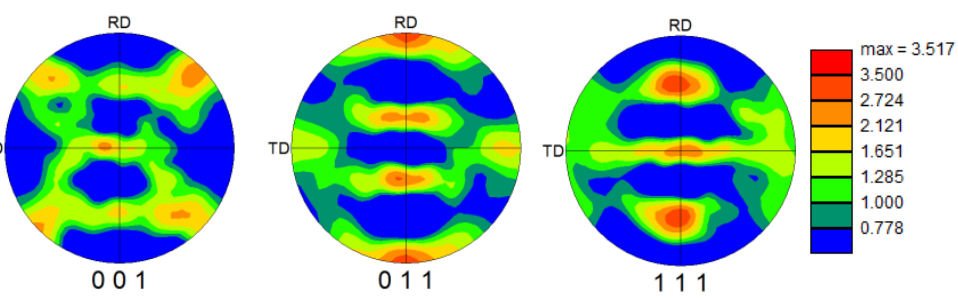

Fig. 1. (a) EBSD orientation map with insets of martensite regions showing the initial microstructure of the DP590 steel. (b) EBSD image quality map corresponding to (a) distinguishing martensite (dark) from ferrite. (c) Pole figures showing the initial crystallographic texture of the DP590 steel.

\subsection{Material testing}

\subsubsection{Simple tension}

Simple tension specimens according to the ASTM E-8 standard, with a uniform section of 75 $\mathrm{mm}$, gage length of $50 \mathrm{~mm}$ and width of $12.5 \mathrm{~mm}$ were machined from the DP590 sheet with the loading direction aligned with the sheet rolling direction (RD). The experiments were performed on an MTS Landmark 370 servohydraulic testing machine, at a nominal strain rate of $5 \times 10^{-4} / \mathrm{s}$. During the experiments, the strain was acquired using the VIC-2D Digital Image Correlation (DIC) system using a 2 MPixel camera with $35 \mathrm{~mm}$ lenses. The DIC measurements were compared with those measured by a mechanical extensometer and found to be identical. The obtained flow curve is shown in Fig. 2. 


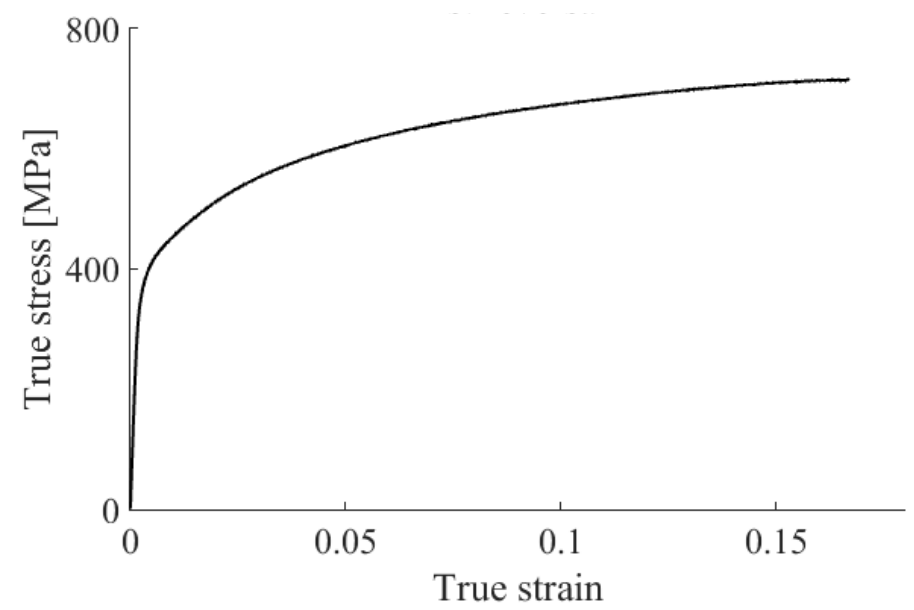

Fig. 2. True stress-true strain curve of DP590 in the rolling direction.

\subsubsection{Cyclic tension-compression-tension testing}

Cyclic tension-compression-tension experiments were performed with the specimen geometry shown in Fig. 3. The testing setup and machine used to perform the test are depicted in Fig. 4 (Kuwabara et al., 2009; Kuwabara et al., 2001; Verma et al., 2011). The machine consists of two pairs of comb shaped dies that mesh and can move in the axial direction relative to each other, while at the same time providing lateral support to the thin specimen to avoid buckling. During testing, the axial displacement is applied by cylinder A in Fig. 4b, while the die clamping force is maintained constant by cylinder $\mathrm{B}$. The clamping force induced a lateral pressure on the specimen of $5.4 \mathrm{MPa}$, or less than $2 \%$ of the material yield stress. The strain was measured with a YFLA-2 (Tokyo Sokki Kenkyujo) high-elongation strain gage. The specimens were lubricated with a sandwich of Vaseline and $0.05 \mathrm{~mm}$ Teflon sheets. Before the experiments, the machine was run without a specimen, to calibrate for the friction present. The friction coefficient was determined to be approximately equal to 0.02 . Both the forward and cyclic experiments were performed thrice and repeatable results were obtained in every case.

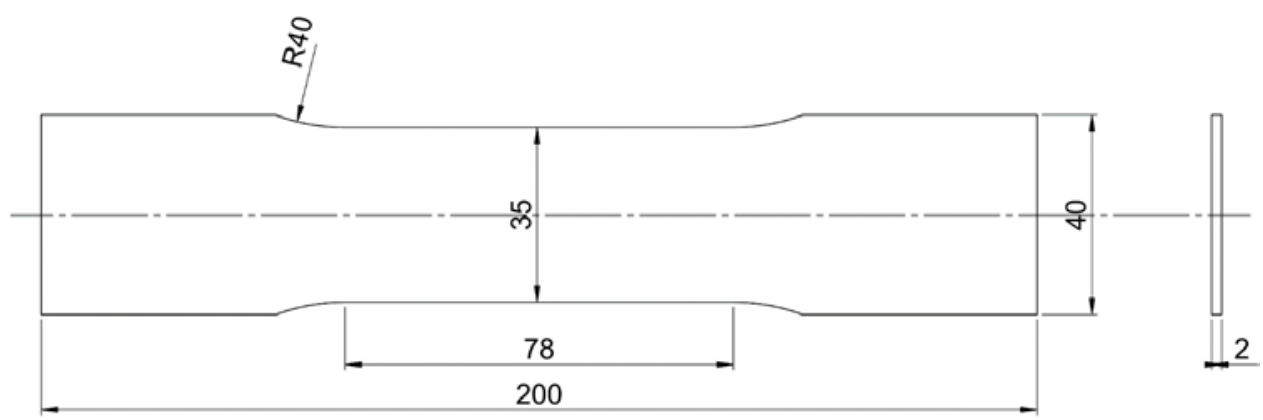

Fig. 3. Specimen dimensions for cyclic tension-compression-tension experiments. 

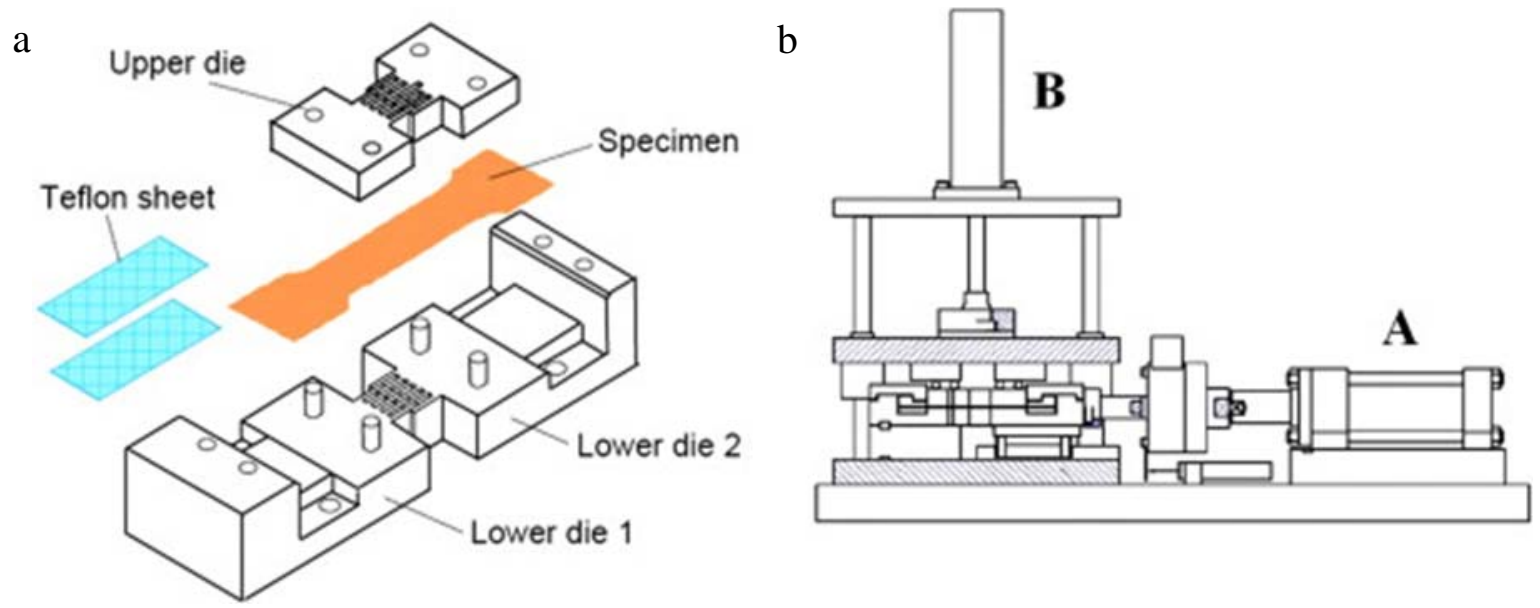

Fig. 4. (a) Cyclic tension-compression testing setup. (b) Overview of the cyclic tensioncompression testing machine with the loading (A) and clamping (B) cylinders identified.

The measured true stress-true strain tension-compression-tension cyclic curves to several strain levels are shown in Fig. 5a. We will refer to the first loading tensile path as the forward tension, to the second compressive loading path as the $1^{\text {st }}$ reversal, and to the third tensile loading path as the $2^{\text {nd }}$ reversal. It is observed that the material exhibits the classical decreasing hardening rate throughout the response in forward tension, which is typical for materials in which the plastic deformation is accommodated by crystallographic slip. Upon unloading, the material exhibits an initial linear portion and a subsequent non-linear portion of unloading behavior (Fig. 5a, b, and c). The non-linear unloading behavior upon the $1^{\text {st }}$ and the $2^{\text {nd }}$ reversal appears to be very similar although the total accumulated plastic strains upon the $2^{\text {nd }}$ reversal is twice as that upon the $1^{\text {st }}$ reversal. Transients upon the $1^{\text {st }}$ reversal (Fig. 5b) are larger than transients upon the $2^{\text {nd }}$ reversal (Fig. 5c). Also, transients are more pronounced with increase in the strain amplitude (Fig. 2b and c). In particular, the comparison of the true stress-true strain cyclic curves during $1^{\text {st }}$ and $2^{\text {nd }}$ reversal reveals that the $2^{\text {nd }}$ reversal curves exhibit significantly shorter transient at lower stresses than the $1^{\text {st }}$ reversal curves (Fig. 5d)). Interestingly, the $0.12^{\text {nd }}$ reversal transient overlaps with the $0.061^{\text {st }}$ reversal transient. Eventually all curves during the $2^{\text {nd }}$ reversal evolve towards the monotonic tension curve measured during forward loading. The permanent softening effect is shown in Fig. 2e. This type of permanent softening after strain reversal was first observed in (Hasegawa et al., 1975) during tension and compression loading and more recently during forward and reverse simple shear loading in (Gracio et al., 2004). This softening was attributed to the annihilation of dislocations (recovery effects) after the reversal of the slip directions during loading in the reverse direction. Normalized hardening rates during the $1^{\text {st }}$ and the $2^{\text {nd }}$ reversal are less steep and show a more gradual change with normalized stress in comparison with that during the forward loading (Fig. 2f). The normalization factor is the shear modulus, $\mu$, taken to be 81.6 GPa (Meyers and Chawla, 2009). The curves start from the value 
corresponding to the ratio between Young's modulus and the shear modulus, $E / \mu$. Upon the $1^{\text {st }}$ and the $2^{\text {nd }}$ reversal, the strain hardening rates are less steep compared to that during the forward tension. The strain hardening rate during continuous straining upon the $1^{\text {st }}$ reversal decrease faster than that during forward tension. As a result, the flow stress exhibits permanent softening (Fig. 5e). Due to non-linear unloading, the reversal curves exhibit lower strain-hardening rates at zero macroscopic stress.

The focus of the present paper is to develop a constitutive law for two-phase materials sensitive to strain reversals. Specifically, the aim is to incorporate the effects described above in a crystallographic hardening model operating within the EPSC homogenization and to apply it to the data sets collected for DP590. The modeling framework is described in the next section.
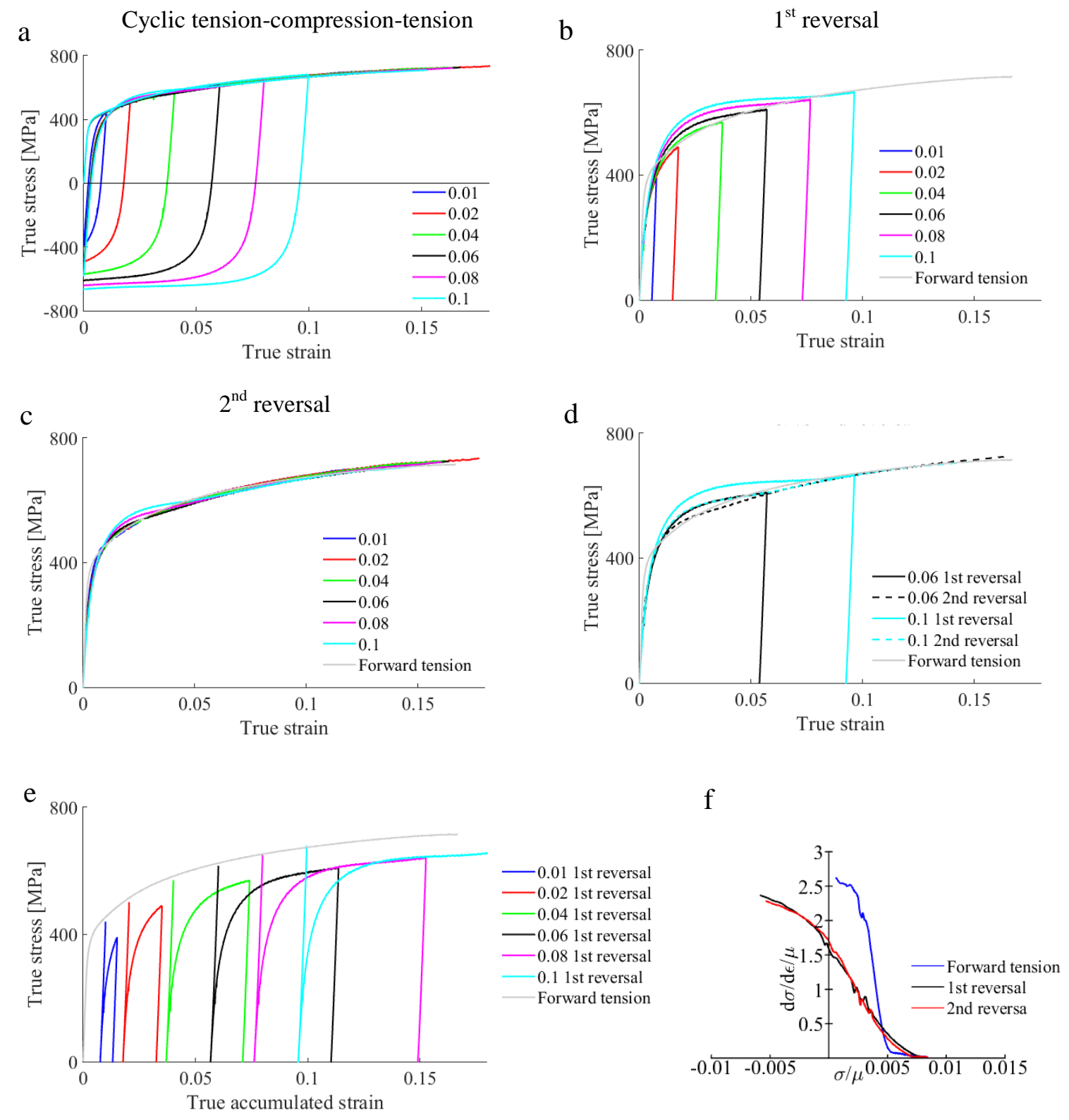
Fig. 5. (a) True stress-true strain cyclic tension-compression-tension curves for DP590 measured along RD. (b) Comparison of the $1^{\text {st }}$ reversal (i.e. compression) curves pre-strained in tension to different strain levels. (c) Comparison of the $2^{\text {nd }}$ reversal (i.e. tension) curves pre-strained in tension followed by compression to different strain levels. (d) Comparison of the $1^{\text {st }}$ and the $2^{\text {nd }}$ reversal curves. (e) Forward and the $1^{\text {st }}$ reversal curves as a function of accumulated true strain showing drops in yield stress i.e. the permanent softening upon load reversal. (f) Normalized strain hardening rate plots during forward loading, and the $1^{\text {st }}$ and the $2^{\text {nd }}$ reverse loadings for the 0.1 strain level curves.

\section{Modeling framework}

The EPSC homogenization originally developed in (Neil et al., 2010; Turner and Tomé, 1994) is used here as a platform to implement the model for cyclic deformation to large plastic strains of two-phase materials. The recently reported particular version of the model (Zecevic and Knezevic, 2015) integrating a hardening law based on evolution of dislocation density and slip system backstresses is extended here to perform the two level homogenization and to handle slip system backstresses in every phase. These backstresses are due to not only grain-to-grain interactions within a single-phase but also among multiple phases, specifically through the effect of GNDs. The effect of backstress due to GNDs is modeled through a coated ellipsoidal inclusion scheme, which also includes the localized hardening.

\subsection{Summary of EPSC}

In EPSC, a polycrystal is treated as a collection of single crystals each having a specific orientation, an ellipsoidal shape, and a volume fraction. Effective properties of the polycrystal are found using the self-consistent scheme, where each crystal is treated as an elasto-plastic inclusion in the homogeneous equivalent medium (HEM), which would have the sought properties of the polycrystal. In this summary, we use “.” to represent a contracted or dot product and “ $\bigotimes$ ” for uncontracted or tensor product.

The macroscopic Jaumann stress rate and strain rate are linked through the following linear relationship:

$$
\widehat{\boldsymbol{\sigma}}=\mathbf{L} \dot{\boldsymbol{\varepsilon}}
$$

where $\mathbf{L}$ is the unknown instantaneous elasto-plastic stiffness tensor of the polycrystal, which is solved iteratively through the standard SC procedure (Turner and Tomé, 1994) satisfying stress equilibrium and strain compatibility (Eshelby, 1957). The relation between a strain rate in individual crystals and the macroscopic strain rate is:

$$
\dot{\boldsymbol{\varepsilon}}^{c}=\mathbf{A}^{c} \dot{\boldsymbol{\varepsilon}},
$$

where $\mathbf{A}^{c}=\left(\mathbf{L}^{c}+\mathbf{L}^{c^{*}}\right)^{-\mathbf{1}}\left(\mathbf{L}^{c^{*}}+\mathbf{L}\right)$ is the localization tensor for elasto-plastic ellipsoidal inclusion where $\mathbf{L}^{c}$ is the instantaneous single crystal, $c$, elasto-plastic stiffness, which will be explained in detail below. $\mathbf{L}^{c^{*}}=\mathbf{L}\left(\mathbf{S}^{c^{-1}}-\mathbf{I}\right)$ is the effective stiffness, which relates the stress and total strain rate in a grain to those in the macroscopic medium through the following 
interaction equation: $\left(\widehat{\boldsymbol{\sigma}}^{c}-\widehat{\boldsymbol{\sigma}}\right)=-\mathbf{L}^{c^{*}}\left(\dot{\boldsymbol{\varepsilon}}^{c}-\dot{\boldsymbol{\varepsilon}}\right) . \mathbf{S}^{c}$ is the symmetric Eshelby tensor and $\mathbf{I}$ is the fourth rank identity matrix. Using the condition that the polycrystal stress and strain rate is equal to the volume average of the stress and strain rate in grains:

$$
\widehat{\boldsymbol{\sigma}}=\left\langle\widehat{\boldsymbol{\sigma}}^{c}\right\rangle,
$$

and

$$
\dot{\boldsymbol{\varepsilon}}=\left\langle\dot{\boldsymbol{\varepsilon}}^{c}\right\rangle,
$$

the equation for $\mathbf{L}$ becomes:

$$
\mathbf{L}=\left\langle\mathbf{L}^{c} \mathbf{A}^{c}\right\rangle\left\langle\mathbf{A}^{c}\right\rangle^{-\mathbf{1}} .
$$

The Cauchy stress rate is related to Jaumann rate: $\dot{\boldsymbol{\sigma}}=\widehat{\boldsymbol{\sigma}}+\left\langle\mathbf{W}^{\mathrm{c}} \boldsymbol{\sigma}^{\mathrm{c}}\right\rangle-\left\langle\boldsymbol{\sigma}^{\mathrm{c}} \mathbf{W}^{\mathrm{c}}\right\rangle=\mathbf{L} \dot{\boldsymbol{\varepsilon}}+\left\langle\mathbf{W}^{\mathrm{c}} \boldsymbol{\sigma}^{\mathrm{c}}\right\rangle-$ $\left\langle\boldsymbol{\sigma}^{\mathrm{c}} \mathbf{W}^{\mathrm{c}}\right\rangle$, where $\mathbf{W}^{\mathrm{c}}$ is elastic spin of crystal, $c$, defined in section 3.4. The relation is integrated explicitly over time of applied deformation process to calculate the macroscopic Cauchy stress. After $\mathbf{L}$ has been successfully calculated at a time instance, $n$, the field variables are updated with the solution achieved at the current increment e.g. $\boldsymbol{\sigma}_{n+1}=\boldsymbol{\sigma}_{n}+\mathbf{L}_{n} \dot{\boldsymbol{\varepsilon}}_{n} \Delta t+\left\langle\mathbf{W}_{n}^{c} \boldsymbol{\sigma}_{n}^{c}\right\rangle \Delta t-$ $\left\langle\boldsymbol{\sigma}_{n}^{c} \mathbf{W}_{n}^{c}\right\rangle \Delta t$.

At the single crystal level, the constitutive relation between the Jaumann stress rate, $\widehat{\boldsymbol{\sigma}}^{c}$, and the strain rate, $\dot{\boldsymbol{\varepsilon}}^{c}$, is:

$$
\widehat{\boldsymbol{\sigma}}^{c}=\mathbf{C}^{c}\left(\dot{\boldsymbol{\varepsilon}}^{c}-\sum_{\mathrm{s}} \mathbf{m}^{c, s} \dot{\gamma}^{c, s}\right)-\boldsymbol{\sigma}^{c} \operatorname{tr}\left(\dot{\boldsymbol{\varepsilon}}^{c}\right),
$$

where $\mathbf{C}^{c}$ is the elastic stiffness tensor of single crystal and $\sum_{\mathbf{s}} \mathbf{m}^{c, s} \dot{\gamma}^{c, s}$ is the plastic strain rate defined as the sum of shear strain rates, $\dot{\gamma}^{c, s}$, from each slip system, s. In Eq. (6), $\mathbf{m}^{c, s}=$ $0.5\left(\mathbf{b}^{c, s} \otimes \mathbf{n}^{c, s}+\mathbf{n}^{c, s} \otimes \mathbf{b}^{c, s}\right)$ is the symmetric portion of the Schmid tensor of the slip system $s$, where, $\mathbf{b}^{c, s}$ and $\mathbf{n}^{c, s}$ are orthonormal unite vectors representing the slip direction and slip plane normal, respectively. The elastic stiffness is calculated at the beginning of each deformation increment meaning that textural changes are accounted for. The invariant single crystal elastic constants are taken to be: $C_{11}=233,000 \mathrm{MPa}, C_{12}=135,000 \mathrm{MPa}$, and $C_{44}=117,000 \mathrm{MPa}$ for the ferrite phase and the martensite phase (Simmons and Wang, 1971).

For a slip system to be active, the following two conditions must be satisfied: (1) $\mathbf{m}^{c, s} \cdot \boldsymbol{\sigma}^{c}-$ $\tau_{b s}^{c, s}=\tau_{c}^{c, s}$ meaning that the resolved shear stress reduced by the value of backstress, $\tau_{b s}^{c, s}$, reaches the value of slip resistance, $\tau_{c}^{c, s}$ and (2) $\mathbf{m}^{c, s} \cdot \widehat{\boldsymbol{\sigma}}^{c}-\dot{\tau}_{b s}^{c, s}=\dot{\tau}_{c}^{c, s}$, meaning that the stress has to remain on the single crystal yield surface, which evolves due to hardening. The adopted conditions do not allow negative shear to happen on a given slip system, and thereby, each slip system is split into two slip systems having the same plane normal but opposite sense of shearing.

The rates of slip resistance, $\dot{\tau}_{c}^{c, s}$, and backstress, $\dot{\tau}_{b s}^{c, s}$, are linked with shear rates, $\dot{\gamma}^{c, s}$ through following relations (Zecevic and Knezevic, 2015):

$$
\dot{\tau}_{c}^{c, s}=\sum_{s^{\prime}} h^{s s^{\prime}} \dot{\gamma}^{c, s^{\prime}},
$$




$$
\dot{\tau}_{b s}^{c, s}=\sum_{s^{\prime}} h_{b s}^{s s^{\prime}} \dot{\gamma}^{c, s^{\prime}}
$$

where $h^{s s^{\prime}}$ and $h_{b s}^{s s}$ are the hardening matrix and the backstress matrix, respectively. Expressions for $h^{s s^{\prime}}$ and $h_{b s}^{s s^{\prime}}$ follow from the adopted hardening and backstress evolution laws both given in (Zecevic and Knezevic, 2015).

Finally, the linear relationship between the Jaumann rate for a crystal and the strain rate is:

$$
\widehat{\boldsymbol{\sigma}}^{c}=\mathbf{L}^{c} \dot{\boldsymbol{\varepsilon}}^{c},
$$

where $\mathbf{L}^{c}$ is:

$$
\mathbf{L}^{c}=\mathbf{C}^{c}-\mathbf{C}^{c} \sum_{s} \mathbf{m}^{c, s} \otimes\left(\sum_{s^{\prime}}\left(X^{s s^{\prime}}\right)^{-1} \mathbf{m}^{c, s^{\prime}}\left(\mathbf{C}^{c}-\boldsymbol{\sigma}^{c} \otimes \mathbf{i}\right)\right)-\boldsymbol{\sigma}^{c} \otimes \mathbf{i},
$$

with:

$$
X^{s s^{\prime}}=h^{s s^{\prime}}+h_{b s}^{s s^{\prime}}+\mathbf{C}^{c} \cdot \mathbf{m}^{c, s} \otimes \mathbf{m}^{c, s^{\prime}} .
$$

\subsection{Incorporation of the effects of GNDs accumulated around a hard phase}

Overall properties of composite microstructures are usually higher than the properties obtained based on the mixture-rule of constituent phases. In metallic composite microstructures such phenomena stems from localized hardening mechanisms arising from, for example, dislocation substructures. Specifically, GNDs are known to accumulate around a harder phase present in the microstructure, causing localized hardening. In case of DP steels, the measured data indicate that the population of GNDs close to the ferrite-martensite phase boundaries are approximately one order of magnitude higher than inside the ferrite grains (Kadkhodapour et al., 2011; Korzekwa et al., 1984). A high content of GNDs present in the vicinity of martensite are a consequence of strains produced by the austenite-martensite transformation to maintain lattice continuity and strains accumulated during subsequent plastic deformation. Additionally, the measured data shows that the hardness of the interface regions gradually increase from the ferrite to the martensite phase over a thickness of approximately $1.5 \mu \mathrm{m}$ (Kadkhodapour et al., 2011). To take into account these localized hardening effects for modeling of DP590 in EPSC, we adapt the micromechanical approach of 'coated ellipsoidal inclusions' presented in (Berbenni and Cherkaoui, 2010). The adopted approach is similar to the one introduced in (Kadkhodapour et al., 2011), where an FE unit cell was used to model the martensite phase reinforced by a layer around it in a macroscopic sense. The constitutive response was based on the J2 plasticity. Here, the analytical thin layer approximation is used. To this end, we adopt the analytical solution to the interlayer, where every layer/grain/region follows the crystal plasticity constitutive response. The analytical solution employed here calculates the average strain rate within the layer and the grain/region while neglecting any gradients of the field variables. Additionally, the analytical

solution is based on an approximation in terms of relating the average strain rate in the layer and the average strain rate in the inclusion, which is valid in the case of a homothetic spherical inclusion and associated thin layer. The approximation facilitates solving the coated inclusion 
problem in a compact and computationally efficient way. The approach was originally referred as the homothetic spherical inclusion with coating and the solution to the problem within an infinite matrix under remotely applied load has been presented in (Cherkaoui et al., 1995; Taupin et al., 2010; Taupin et al., 2013). The coated layer around the martensite regions is modeled here as reinforcement i.e. it is harder than the ferrite grains within the HEM facilitating the martensite region to have a higher state of stress than it would otherwise be possible by direct embedding the martensite grains in the HEM. In addition to being the source of local hardening, GNDs around the martensite phase are a source of backstresses (Nesterova et al., 2015; Taupin et al., 2013). These effects are also incorporated in the model, as will be discussed in detail in section 3.6.

Figure 6 schematically shows a ferrite grain and a martensite region surrounded by the reinforcement layer of GNDs embedded within the HEM. Expressions for the localization tensors developed in (Berbenni and Cherkaoui, 2010) are adapted for the case of one inclusion (the martensite region) with coating (the layer with GNDs) as:

$$
\begin{aligned}
& \mathbf{A}^{m r}=\boldsymbol{\alpha}^{m r}\left[\mathbf{I}+\mathbf{T}(\mathbf{L})\left[\phi_{m r}\left(\mathbf{L}^{m r}-\mathbf{L}\right) \boldsymbol{\alpha}^{m r}+\phi_{l}\left(\mathbf{L}^{l}-\mathbf{L}\right) \boldsymbol{\alpha}^{l}\right]\right]^{-1}, \\
& \mathbf{A}^{l}=\boldsymbol{\alpha}^{l}\left[\mathbf{I}+\mathbf{T}(\mathbf{L})\left[\phi_{m r}\left(\mathbf{L}^{m r}-\mathbf{L}\right) \boldsymbol{\alpha}^{m r}+\phi_{l}\left(\mathbf{L}^{l}-\mathbf{L}\right) \boldsymbol{\alpha}^{l}\right]\right]^{-1},
\end{aligned}
$$

where: $\boldsymbol{\alpha}^{m r}=\left[\mathbf{I} \phi_{m r}+\phi_{\mathrm{l}}\left(\mathbf{I}-\mathbf{T}\left(\mathbf{L}^{l}\right)\left(\mathbf{L}^{l}-\mathbf{L}^{m r}\right)\right)\right]^{-1}$ and $\boldsymbol{\alpha}^{l}=\left[\mathbf{I}-\mathbf{T}\left(\mathbf{L}^{l}\right)\left(\mathbf{L}^{l}-\mathbf{L}^{m r}\right)\right] \boldsymbol{\alpha}^{m r}$. The relative fraction of the martensite region in the composite inclusion is $\phi_{m r}=\frac{f_{m r}}{f_{m r}+f_{l}}$ and analogously, the relative fraction of the layer in the composite inclusion is $\phi_{l}=\frac{f_{l}}{f_{m r}+f_{l}}$. Tensors of $4^{\text {th }}$ rank, $\mathbf{L}, \mathbf{L}^{l}$ and $\mathbf{L}^{m r}$ are instantaneous elasto-plastic stiffness tensors for the HEM, the layer of GNDs, and the martensite grains, respectively. For the homothetic ellipsoidal inclusions with a thin layer the following relations apply: $\mathbf{T}\left(\mathbf{L}^{l}\right)=\mathbf{S}\left(\mathbf{L}^{l}\right) \mathbf{L}^{l^{-\mathbf{1}}}$ and $\mathbf{T}(\mathbf{L})=\mathbf{S}(\mathbf{L}) \mathbf{L}^{-\mathbf{1}}$, where $\mathbf{T}\left(\mathbf{L}^{l}\right)$ and $\mathbf{T}(\mathbf{L})$ are $4^{\text {th }}$ rank symmetric tensors derived from the symmetric Eshelby tensors $\mathbf{S}\left(\mathbf{L}^{l}\right)$ and $\mathbf{S}(\mathbf{L})$, associated with the elasto-plastic instantaneous stiffness tensors $\mathbf{L}$ and $\mathbf{L}$.

The self-consistent equation, Eq. (5), can now be evaluated with appropriate localization tensors either $\mathbf{A}^{c, f}=\left(\mathbf{L}^{c, f}+\mathbf{L}^{c, f^{*}}\right)^{-\mathbf{1}}\left(\mathbf{L}^{c, f^{*}}+\mathbf{L}\right)$ for ferrite grains or $\mathbf{A}^{c, m r}$ for the martensite region or $\mathbf{A}^{c, l}$ for the layer depending on where the given grain belongs. The last two have been defined in Eqs. (12) and (13), respectively.

In summary, the developed model achieves the interaction between the martensite single crystal or polycrystalline regions and the overall HEM through a thin reinforcement layer. The interaction through the layer permits stress in the martensite to be of higher magnitude than without the layer. 


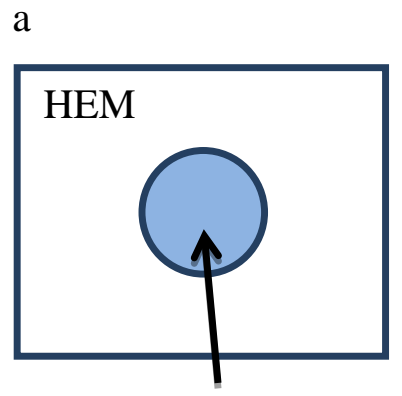

Ferrite grain

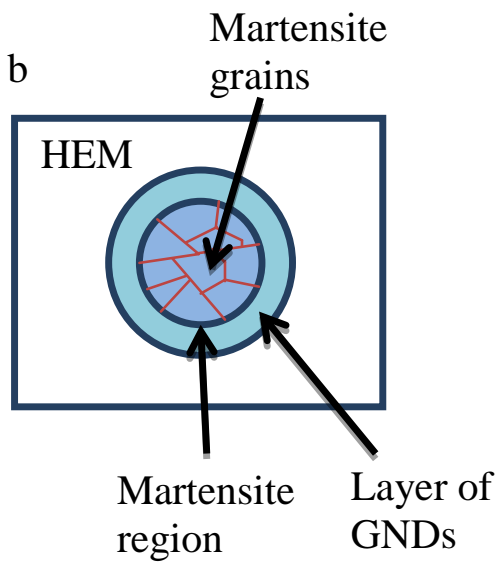

Fig. 6. Schematics showing (a) a ferrite grain embedded within the homogeneous equivalent medium (HEM) representing the average behavior of the aggregate and (b) martensite polycrystalline region surrounded by a layer of GNDs embedded within the HEM for modeling of DP590 microstructure.

\subsection{Two-level SC homogenization}

Martensite regions usually consist of multiple martensite grains (Fig. 1a). The strength of the interactions between relatively hard martensite grains is different from that between relatively soft ferrite grains. To better capture the interactions between grains within the martensite regions, the $2^{\text {nd }}$ level SC homogenization is introduced. Here individual response of martensite grains enters the $2^{\text {nd }}$ level HEM, which is response of the martensite region comprised of the martensite grains. The algorithm for $2^{\text {nd }}$ level homogenization is analogous to that outlaid in section 3 for the $1^{\text {st }}$ level of homogenization and consists of the following steps:

Step 1: An applied strain rate over the martensite region is defined through the localization tensor, $\mathbf{A}^{m r}$, for a martensite region:

$$
\dot{\boldsymbol{\varepsilon}}^{m r}=\mathbf{A}^{m r} \dot{\boldsymbol{\varepsilon}}
$$

where $\dot{\boldsymbol{\varepsilon}}$ is macroscopic strain rate. Equation (14) for a martensite region is analogous to Eq. (2) for a ferrite grain with the difference that an appropriate localization tensor for the martensite region, defined with Eq. (12), is used.

Step 2: The instantaneous elasto-plastic stiffness tensor of a martensite grain, $\mathbf{L}^{c, m}$, follows Eq. (10). The Jaumann stress rate for a grain from the martensite region is then:

$$
\widehat{\boldsymbol{\sigma}}^{c, m}=\mathbf{L}^{c, m} \dot{\boldsymbol{\varepsilon}}^{c, m}=\mathbf{L}^{c, m} \mathbf{A}^{c, m} \dot{\boldsymbol{\varepsilon}}^{m r}
$$

where $\mathbf{A}^{c, m}=\left(\mathbf{L}^{c, m}+\mathbf{L}^{m r}\left(\mathbf{S}^{c, m-1}-\mathbf{I}\right)\right)^{-\mathbf{1}}\left(\mathbf{L}^{m r}\left(\mathbf{S}^{c, m-1}-\mathbf{I}\right)+\mathbf{L}^{m r}\right)$ is the localization tensor for an ellipsoidal inclusion representing a martensite grain embedded in the martensite region with the instantaneous elasto-plastic stiffness tensor, $\mathbf{L}^{m r}$. Equation (15) includes the relationship between the applied strain rate on the martensite region, $\dot{\boldsymbol{\varepsilon}}^{m r}$, and the strain rate in an individual crystal of martensite, $\dot{\boldsymbol{\varepsilon}}^{c, m}$. The stress rate is used to calculate the resolved shear stress rate and ensure active slip systems in a given martensite grain. 
Step 3: A SC estimate of the martensite region's instantaneous elasto-plastic stiffness tensor, $\mathbf{L}^{m r}$ , is evaluated with the applied strain rate, $\dot{\boldsymbol{\varepsilon}}^{m r}$, defined in step 1 and single crystal properties of martensite defined in step 2, according to self-consistent equation:

$$
\mathbf{L}^{m r}=\left\langle\mathbf{L}^{c, m} \mathbf{A}^{c, m}\right\rangle\left\langle\mathbf{A}^{c, m}\right\rangle^{-\mathbf{1}} .
$$

In order to calculate the localization tensor for a martensite region, $\mathbf{A}^{m r}$, and eventually the instantaneous elasto-plastic stiffness of that region, $\mathbf{L}^{m r}$, the overall instantaneous elasto-plastic stiffness tensor must be known, which is itself a function of the instantaneous elasto-plastic stiffness of martensite region, $\mathbf{L}^{m r}$. Therefore SC equations at the $1^{\text {st }}$ and the $2^{\text {nd }}$ level must be solved simultaneously.

The equation for the SC instantaneous elasto-plastic stiffness solved at both homogenization levels has the same form of $\mathbf{L}^{s c}=\left\langle\mathbf{L}^{c} \mathbf{A}^{c}\right\rangle\left\langle\mathbf{A}^{c}\right\rangle^{-\mathbf{1}}$. At the $1^{\text {st }}$ level of homogenization, the solution is the instantaneous elasto-plastic stiffness of the polycrystal, $\mathbf{L}^{s c}=\mathbf{L}$, while at the $2^{\text {nd }}$ level the solution is the instantaneous elasto-plastic stiffness of the martensite region, $\mathbf{L}^{s c}=\mathbf{L}^{m r}$. The starting guess for $\mathbf{L}$ is a converged value from a previous increment. For each iteration of the $1^{\text {st }}$ level homogenization, the $2^{\text {nd }}$ level homogenization is performed and $\mathbf{L}^{s c}=\mathbf{L}^{m r}$ is calculated for each martensite region starting from the $\mathbf{L}^{m r}$ available from a previous iteration. Iterations are performed until both $\mathbf{L}$ and $\mathbf{L}^{m r}$ stop changing to a prescribed tolerance value i.e. until the difference between a previous iteration solution and a current iteration solution is less than a value of prescribed tolerance (taken here to be $0.1 \%$ ).

\subsection{Texture evolution}

For completeness, we briefly reflect on the crystallographic texture evolution calculations in the two-level EPSC model. The lattice rotation rate tensor, $\mathbf{W}^{c}$, is defined as:

$$
\mathbf{W}^{c}=\mathbf{W}^{a p p}+\Pi^{c}-\mathbf{W}^{p, c}
$$

where $\mathbf{W}^{a p p}$ is the applied rotation rate, $\boldsymbol{\Pi}^{\mathbf{c}}$ is the contribution coming from the antisymmetric part of the Eshelby tensor (Lebensohn and Tomé, 1993), and $\mathbf{W}^{p, c}$ is the plastic rotation rate. The plastic rotation rate is calculated from the shearing rates as:

$$
\mathbf{W}^{p, c}=\sum_{s} \mathbf{q}^{c, s} \dot{\gamma}^{c, s}
$$

where $\mathbf{q}^{c, s}=0.5\left(\mathbf{b}^{c, s} \otimes \mathbf{n}^{c, s}-\mathbf{n}^{c, s} \otimes \mathbf{b}^{c, s}\right)$ is the anti-symmetric portion of the dyadic product of the two unit vectors, the Burgers direction and the slip plane normal. Equation (17) is used for the evolution of crystal orientations for both ferrite and martensite grains. We evolve the crystal orientation of the layer using the applied spin and the plastic spin, $\mathbf{W}^{c, l}=\mathbf{W}^{a p p}-\mathbf{W}^{p, c}$.

\subsection{Hardening law}

In the description that follows, the Greek superscript $\alpha$ denotes slip modes known to operate in both ferrite and martensite: $\alpha=1$ for $\{110\}\langle 1 \overline{1} 1\rangle$ and $\alpha=2$ for $\{112\}\langle 11 \overline{1}\rangle$ while the superscripts $s, s$ ' span over the individual slip systems belonging to the modes. Additionally, the slip systems have positive $s+$ and negative $s$ - directions, which are arbitrarily chosen. 
Hardening of slip systems is based on the rate of dislocation storage, which is a thermally activated mechanism and, hence, it is dependent on temperature and also on strain rate. In several prior studies, a similar hardening model has been successfully applied to a range of metals involving different crystal structure: hexagonal close packed (HCP) Zr (Beyerlein and Tomé, 2008; Knezevic et al., 2013b; Knezevic and Landry, 2015; Knezevic et al., 2014e; Knezevic et al., 2015), Be (Brown et al., 2012; Knezevic et al., 2013a; Zecevic et al., 2015a), Mg (Beyerlein et al., 2011; Lentz et al., 2015a; Lentz et al., 2015b), body centered cubic (BCC) Ta (Ardeljan et al., 2014a; Ardeljan et al., 2015a; Ardeljan et al., 2014b; Bhattacharyya et al., 2015; Knezevic et al., 2014a; Knezevic et al., 2014e), face centered cubic (FCC) alloys (Knezevic et al., 2014b; Zecevic and Knezevic, 2015), and orthorhombic uranium (Ardeljan et al., 2015b; Knezevic et al., 2012; Knezevic et al., 2013d; Knezevic et al., 2013e). In this model, the slip resistance is defined as:

$$
\tau_{c}^{s}=\tau_{0}^{\alpha}+\tau_{f o r}^{s}+\tau_{d e b}^{\alpha},
$$

where $\tau_{0}^{\alpha}$ is an initial value of the slip resistance that includes the Peierls stress and the HallPetch-like grain size barrier contribution, $\tau_{\text {for }}^{S}$ is a forest term dependent on the statistically stored dislocations and $\tau_{d e b}^{\alpha}$ is a debris term dependent on the amount of dislocations stored as organized debris. The forest term contribution to the evolution of slip resistance is given by a Taylor-type relation which includes the latent hardening term (Kitayama et al., 2013):

$$
\tau_{\text {for }}^{s}=b^{\alpha} \chi \mu^{\alpha} \sqrt{\rho_{\text {tot }}^{s}+L \sum_{s^{\prime}} \rho_{\text {tot }}^{s^{\prime}}},
$$

where $b^{\alpha}=2.4810^{-10} \mathrm{~m}$ is the Burgers vector of slip mode, $\chi=0.9$ is a dislocation interaction constant, $\rho_{\text {tot }}^{s}$ is the total forest dislocation density for $s^{\text {th }}$ slip system $(s \in \alpha)$ and $L$ is a latent hardening parameter, taken here to be 1.05 . The contribution to slip resistance due to the dislocation debris is based on an extended Taylor-type relation (Madec et al., 2003):

$$
\tau_{d e b}^{\alpha}=k_{d e b} \mu^{\alpha} b^{\alpha} \sqrt{\rho_{d e b}} \log \left(\frac{1}{b^{\alpha} \sqrt{\rho_{d e b}}}\right),
$$

where $k_{d e b}=0.086$ is a material independent constant that recovers the Taylor law i.e. Eq. (20) for low values of dislocation density and $\rho_{d e b}$ is the density of dislocations stored as debris.

Here, we adapt the evolution laws presented in (Zecevic and Knezevic, 2015) to simulate hardening during strain path changes of DP Steel. The underlying idea for introducing a deformation-history-dependent directionality in a dislocation-based hardening law is based on introducing a fraction of reversible stored dislocations accumulated during forward loading. To this end, each slip system is split in two systems i.e. $s^{+}$and $s^{-}$having the same slip plane but opposite directions. Therefore, the total dislocation density on each slip system is written as the following sum:

$$
\rho_{\text {tot }}^{s}=\rho_{\text {for }}^{s}+\rho_{\text {rev }}^{s^{+}}+\rho_{\text {rev }}^{s^{-}},
$$


where $\rho_{\text {for }}^{s}$ is the forward dislocation density common to both directions $s^{+}$and $s^{-}$and $\rho_{\text {rev }}^{s^{+}}$and $\rho_{\text {rev }}^{s^{-}}$are the reversible dislocation densities associated with the $s^{+}$and $s^{-}$directions, respectively. These dislocation populations follow different physically-based evolution laws that depend on the previous strain history and shearing direction. The forward dislocations evolution follows the following law (Kocks and Mecking, 1981):

$$
\frac{\partial \rho_{\text {for }}^{s}}{\partial \gamma^{s}}=(1-p) k_{1}^{\alpha} \sqrt{\rho_{\text {for }}^{s}+\rho_{\text {rev }}^{s}}-k_{2}^{\alpha}(\dot{\varepsilon}, T) \rho_{\text {for }}^{s},
$$

where $k_{1}^{\alpha}$ is a coefficient accounting for the rate of generation of statistically stored dislocations, $k_{2}^{\alpha}$ is a rate-sensitive coefficient for dynamic recovery (Beyerlein and Tomé, 2008), and $p$ is a reversibility parameter having value between 0 and $1 . \rho_{\text {rev }}^{s}$ is the dislocation density associated with the active shearing direction (e.g. if $d \gamma^{s^{+}}>0$ then $\rho_{\text {rev }}^{s}=\rho_{\text {rev }}^{s^{+}}$, and if $d \gamma^{s^{-}}>0$ then $\rho_{\text {rev }}^{s}=\rho_{\text {rev }}^{s-}$ ). The reversibility parameter divides the increment in total dislocation density storage $\left(k_{1}^{\alpha} \sqrt{\rho_{\text {for }}^{s}+\rho_{\text {rev }}^{s}} d \gamma^{s}\right)$ into a forward $\left((1-p) k_{1}^{\alpha} \sqrt{\rho_{\text {for }}^{s}+\rho_{\text {rev }}^{s}} d \gamma^{s}\right)$ and a reversible $\left(p k_{1}^{\alpha} \sqrt{\rho_{\text {for }}^{s}+\rho_{\text {rev }}^{s}} d \gamma^{s}\right)$. The reversible dislocation populations are understood as loosely tangled dislocations that can glide in opposite direction upon load reversal. When the content of dislocation substructures is low, the content of reversible dislocations within the total dislocation content is expected to be large, meaning that the reversibility parameter should take values of close to unity. The buildup of dislocation substructures with the continuation of plastic straining will obstruct dislocation motion in the reverse direction as well as the overall population of dislocations that are loosely tangled will decrease. At this point, the reversibility parameter should take valued lower that unity. The dislocation debris significantly obstructing the reversible motion of dislocations was found to develop in the microstructure at a strain of approximately 0.4 (Kitayama et al., 2013). Since the accumulated strain in all simulations performed here is much less than 0.4 , it is reasonable to adopt unity for the value of the reversibility parameter (Kitayama et al., 2013; Zecevic and Knezevic, 2015). The increment in total dislocation density, $k_{1}^{\alpha} \sqrt{\rho_{\text {for }}^{s}+\rho_{\text {rev }}^{s}} d \gamma^{s}$, depends on the forward dislocation density, $\rho_{\text {for }}^{s}$, and the reversible dislocation density associated with active shear direction, $\rho_{\text {rev }}^{s}$, which differs from the original implementation in (Zecevic and Knezevic, 2015) where it depended on the total dislocation density. The rationale for the modification is that dislocations that are being annihilated are not barriers for newly formed dislocations. Evolution of $\rho_{\text {for }}^{S}$ is independent of the direction of shear strain per slip system. The remaining increment in total dislocation density $\left(p k_{1}^{\alpha} \sqrt{\rho_{\text {for }}^{s}+\rho_{\text {rev }}^{s}} d \gamma^{s}\right)$ is stored as reversible dislocation density depending on shear direction on slip system:

(If $d \gamma^{s^{+}}>0$ ) 


$$
\begin{aligned}
& \frac{\partial \rho_{\text {rev }}^{s^{+}}}{\partial \gamma^{s}}=p k_{1}^{\alpha} \sqrt{\rho_{\text {for }}^{s}+\rho_{\text {rev }}^{s}}-k_{2}^{\alpha}(\dot{\varepsilon}, T) \rho_{\text {rev }}^{s^{+}}, \\
& \frac{\partial \rho_{\text {rev }}^{s^{-}}}{\partial \gamma^{s}}=-k_{1}^{\alpha} \sqrt{\rho_{\text {for }}^{s}+\rho_{\text {rev }}^{s}}\left(\frac{\rho_{\text {rev }}^{s^{-}}}{\rho_{0}^{s}}\right)^{m},
\end{aligned}
$$

where $m$ is a parameter controlling rate of dislocation recombination taken here to be 0.5 (Wen et al.) and $\rho_{0}^{s}$ is the total dislocation density present when the shear was reversed on the $s^{\text {th }}$ slip system (Kitayama et al., 2013).

If $d \gamma^{s-}>0$, the increments in $\rho_{\text {rev }}^{s^{-}}$and $\rho_{\text {rev }}^{s^{+}}$evolve in analogues manner to that presented in Eqs. (24) and (25), respectively. The initial conditions for the presented dislocation density evolution laws are: $\rho_{\text {for }}^{s}\left(\gamma^{s}=0\right)=\rho_{\text {initial }}^{s}, \rho_{\text {rev }}^{s^{+}}\left(\gamma^{s}=0\right)=0$ and $\rho_{\text {rev }}^{s^{-}}\left(\gamma^{s}=0\right)=0$. The initial dislocation density was set to $10^{12} \mathrm{~m}^{-2}$ for the ferrite phase and $10^{13} \mathrm{~m}^{-2}$ for the martensite phase.

The coefficient $k_{2}^{\alpha}$ is calculated using:

$$
\frac{k_{2}^{\alpha}}{k_{1}^{\alpha}}=\frac{\chi b^{\alpha}}{g^{\alpha}}\left(1-\frac{k_{B} T}{D^{\alpha}\left(b^{\alpha}\right)^{3}} \ln \left(\frac{\dot{\varepsilon}}{\dot{\varepsilon}_{0}}\right)\right),
$$

where, $k_{B}, \dot{\varepsilon}_{0}, g^{\alpha}$ and $D^{\alpha}$ are constants. They are the Boltzmann constant, a reference strain rate taken to be $10^{7} \mathrm{~s}^{-1}$, an effective activation enthalpy and a drag stress, respectively. Finally, the rate of debris development is coupled to the rate of recovery of all active dislocations through:

$$
d \rho_{d e b}=\sum_{s} q^{\alpha} b^{\alpha} \sqrt{\rho_{d e b}} \frac{\partial \rho_{r e m, t o t}^{s}}{\partial \gamma^{s}}\left|d \gamma^{s^{\prime}}\right|,
$$

where $q^{\alpha}$ is a dislocation recovery rate constant that extracts a fraction of $\alpha$-type dislocations that do not annihilate but become debris from those that do annihilate. Note that the rate of debris generation is proportional to the total dislocation density, i.e. $\frac{\partial \rho_{r e m}^{S}, t o t}{\partial \gamma^{s}}=k_{2}^{\alpha}(\dot{\varepsilon}, T) \rho_{\text {tot }}^{S}$ (Kitayama et al., 2013).

Evidence exist that the dislocations formed during forward straining are more easily annihilated during reversal in DP steel than in single-phase materials, producing a strong Bauschinger effect (Gardey et al., 2005; Wilson and Bate, 1986). In experimental measurements this is seen as decrease in hardness as well as shrinkage of the diffraction peak widths (Wilson and Bate, 1986). Upon strain reversal, generation of dislocations is temporarily retarded because there are loosely-tangled stored dislocations that can easily glide in the backward direction (Beyerlein, 2008). These evidences suggest that a decrease of dislocation density upon strain reversal is another mechanism needed to be incorporated in the model along with evolution laws for different dislocation populations. Therefore, a fraction of stored dislocation is instantaneously annihilated upon strain reversals. 


\subsection{Evolution of backstresses in two-phase materials}

Plastic deformation of DP steels starts by the ferrite phase deforming plastically, while the martensite phase remains elastic (Gong et al., 2016). During this process, GNDs are accumulating around the martensite regions, forming a strain gradient. As mentioned earlier, the GNDs in these regions are a source of localized hardening and are causing backstresses in both the ferrite and the martensite (Nesterova et al., 2015). Additional backstress field is present in the ferrite phase grains arising from heterogeneous dislocation debris distribution.

Physically, the stress field arising from GNDs will cause traction acting on surfaces of martensite regions, increasing the stress within the martensite regions. The stress field in the ferrite matrix around a martensite region will vary with distance from the martensite. The overall volume average stress in a sample at equilibrium is zero. Therefore there is a non-zero value of backstress field present in the ferrite phase as well as a non-zero value of backstress present in the martensite phase entering the volume average stress (Brown and Stobbs, 1971). These individual values can be calculated provided that backstresses are known in every grain in one phase (Brown and Stobbs, 1971).

During plastic deformation, hard cell walls and soft cell interiors form within individual ferrite grains (Mughrabi, 2001). Concurrently, GNDs are accumulated at the interface between the cell walls and the cell interiors ensuring compatible deformation. These GNDs introduce an internal stress field having opposite signs between the cell interiors and the cell walls. The internal stress acts to increase stress action on the hard cell walls and obstruct the plastic flow in soft cell interiors. Therefore, in addition to backstress from GNDs around the martensite regions, there is a backstress field present inside each ferrite grain (stress type III) effecting the grain scale plastic deformation. The volume average of this backstress field (stress type III) over ferrite grains by definition vanishes (Mughrabi, 2001).

While the GND density is related to the gradient in plastic slip fields (Evers et al., 2004), the backstress is governed by the gradient of GND density. Such formulations have been developed within the full-field crystal plasticity finite element framework for both FCC and BCC materials (Evers et al., 2004; Ma et al., 2006a, b, 2007). Within the mean-field model considered here, the gradients exits only between the grain and the HEM as well as the second level homogenization HEM. To better capture the effect of the GND density, we adopt the reinforcing layer formulation (Fig. 6b.). Here, the gradient of plastic strain and stress field vanish within the layer as well as within the grain. To obtain the gradient of strain field within the layer, the gradient of the integral equations within the layer would have to be evaluated (Lipinski and Berveiller, 1989). The backstress would then be defined based on the gradient of the calculated GND density distribution in the layer. The calculated value of backstress based on the GND distribution in the layer would have to be related to particular grains of ferrite and martensite phases. Due to significant difficulties with such a formulation, a phenomenological approach is adopted in this work to approximate the backstress evolution stages: rapid increase of backstress while martensite is elastic and slow evolution of backstress within ferrite grains due to formation of cells and cell walls. Since the GNDs are not explicitly modeled, the backstress is assumed to 
evolve with plastic strain per crystal giving rise to the kinematic hardening effects. Similar approach has been adopted in (Goh et al., 2003; Harder, 1999; Li et al., 2014; Xu and Jiang, 2004). The advantage of the mean-field model presented here is in its computational efficiency.

The stress field around dislocations is tensorial in nature (Bayley et al., 2006). However, only the shear stress exerted on a slip system on which the dislocation glides enters the backstress definition (Evers et al., 2004; Kim et al., 2012). Such formulation is known as self-internal backstress formulation (Bayley et al., 2006) in which the backstress evolves with the plastic strain on individual slip systems (Beyerlein and Tomé, 2007; Choi et al., 2013; Li et al., 2014; Wollmershauser et al., 2012; Zecevic and Knezevic, 2015). Here, an evolution law for backstress with plastic strain on individual slip systems is adopted for ferrite grains. However, since martensite is not deforming plastically during the process of accumulation of GNDs, evolution of backstress in martensite cannot be performed in the same manner. To account for the backstress in martensite, we rely on the fact that the volume average of backstress always vanishes. Thus, the backstress in martensite is defined based on the backstress in ferrite grains.

In what follows, the evolution laws for the slip system backstresses in ferrite and martensite are described. Here, we extend the EPSC model to account for the effect of intra-granular micro backstresses at the slip system level for two-phase materials.

\subsubsection{Ferrite grains during forward loading}

As mentioned earlier, for a slip system to activate, the following condition must be fulfilled: $\mathbf{m}^{s} \cdot \boldsymbol{\sigma}-\tau_{b s}^{s}=\tau_{c}^{s}$. Clearly, satisfying this condition is controlled in part by the evolution of the backstresses.

The backstress on individual slip system is calculated based on the backstress tensor per crystal. The backstress tensor formulation follows the implementation presented in (Harder, 1999). As defined earlier, the scalar value of shear backstress on an active slip system is assumed to be a function of shear strain on that system and represents the stress from GNDs on that slip system. This shear stress is assumed to be uniform within a grain and can be represented in the global sample frame using dyadic products between the slip system Burgers direction and the slip system normal direction defined in the sample frame:

$$
\boldsymbol{\sigma}_{b s}^{c, s, f}=\left(\mathbf{b}^{c, s} \otimes \mathbf{n}^{c, s}+\mathbf{n}^{c, s} \otimes \mathbf{b}^{c, s}\right) \tau_{b s, s y s}^{s}=2 \mathbf{m}^{c, s} \tau_{b s, s y s}^{s},
$$

where $\boldsymbol{\sigma}_{b s}^{c, s, f}$ is a backstress tensor and $\tau_{b s, s y s}^{s}$ is a backstress on the slip system. The expression on the right of Eq. (28) follows from the Schmid tensor $\left(0.5\left(\mathbf{b}^{c, s} \otimes \mathbf{n}^{c, s}+\mathbf{n}^{c, s} \otimes \mathbf{b}^{c, s}\right)=\mathbf{m}^{c, s}\right)$. Note that the backstress, $\tau_{b s, s y s}^{s}$, acts in the local slip system frame defined with the unit vectors: $\mathbf{b}^{c, s^{+}}, \mathbf{n}^{c, s}$ and $\mathbf{b}^{c, s^{+}} \times \mathbf{n}^{c, s}$. It has two components (i.e. $\mathbf{b}^{c, s} \otimes \mathbf{n}^{c, s}, \mathbf{n}^{c, s} \otimes \mathbf{b}^{c, s}$ ) due to the conservation of angular momentum. The total backstress tensor is a sum of the backstress fields from individual slip systems:

$$
\boldsymbol{\sigma}_{b s}^{c, f}=2 \sum_{s^{\prime}} \mathbf{m}^{c, s^{\prime}} \tau_{b s, s y s}^{s^{\prime}},
$$


where the sum spans over all active slip systems. Finally, the backstress on each slip system within a crystal is defined as a resolved shear stress of the crystal level backstress tensor:

$$
\tau_{b s}^{s}=\mathbf{m}^{c, s} \cdot \boldsymbol{\sigma}_{b s}^{c, f}=\tau_{b s, s y s}^{s}+2 \sum_{s^{\prime}} \mathbf{m}^{c, s} \cdot \mathbf{m}^{c, s^{\prime}} \tau_{b s, s y s}^{s^{\prime}},
$$

where the sum over $s^{\prime}$ spans over all active slip systems and $s^{\prime} \neq s$.

For backstress to evolve, ferrite grains must be deforming plastically. The adopted evolution law for backstress, $\tau_{b s, s y s}^{s^{+}}$, during forward loading follows the relations provided in (Beyerlein and Tomé, 2007; Zecevic and Knezevic, 2015), in which the backstress on each slip system evolves as a function of accumulated shear strain. In the presence of plastic deformation, evolution of backstress in the case of shearing in $s^{+}$direction, $d \gamma^{s^{+}}>0$, is:

$$
\begin{aligned}
& \tau_{b s, s y s}^{s^{+}}=\tau_{b s}^{s a t}\left(1-\exp \left(-v \gamma^{s^{+}}\right),\right. \\
& \tau_{b s, s y s}^{s^{-}}=-A \tau_{b s, s y s}^{s^{+}},
\end{aligned}
$$

where $\tau_{b s, s y s}^{s^{+}}$and $\tau_{b s, s y s}^{s^{-}}$are backstresses in the two opposite directions per slip system, $\tau_{b s}^{s a t}$ is a saturation value for backstresses, A and $v$ are fitting parameters and $\gamma^{s^{+}}$is the accumulated shear strain on the $s^{\text {th }}$ slip system. The parameter A introduces an asymmetric evolution of backstress on a slip system in two opposite slip directions. The parameter is introduced to take into account micro-backstresses present in dislocation pile-ups, which are responsible for non-linear unloading after reversal (Sritharan and Chandel, 1997). The physical meaning of parameter A and its magnitude are discussed in detail in (Zecevic and Knezevic, 2015).

In summary, $\tau_{b s}^{s^{+}}$, acts in the direction which is opposite with respect to the resolved shear stress on $s^{+}$, i.e. $\mathbf{m}^{s^{+}} \cdot \boldsymbol{\sigma}-\tau_{b s}^{s^{+}}=\tau_{c}^{s}$, meaning that $\tau_{b s}^{s^{+}}$lowers the activation stress. Simultaneously, $\tau_{b s}^{s^{-}}$aids the resolved shear stress on the slip system $s^{-}$according to: $\mathbf{m}^{s^{-}} \cdot \boldsymbol{\sigma}-$ $\tau_{b s}^{s^{-}}=\tau_{c}^{s}$. These kinematic hardening effects are the micro plasticity processes responsible for non-linear unloading and the BE.

\subsubsection{Ferrite grains during reverse loading}

During loading in the opposite direction, the system that was active during forward deformation, $s^{+}$, quickly unloads and activates in the opposite direction, $s^{-}$. The evolution of backstress after reversal, $d \gamma^{s^{-}}>0$, is given as a piecewise function of shearing strain:

$$
\begin{aligned}
& \tau_{b s, s y s}^{s^{-}}=-(A+1) \tau_{b s 0}^{s^{+}} \exp \left(-\frac{\gamma^{s^{-}}}{\gamma_{b}}\right)+\tau_{b s 0}^{s^{+}}, \tau_{b s}^{s^{+}}=-\frac{1}{A} \tau_{b s}^{s^{-}} \text {if } \tau_{b s}^{s^{-}}<0, \\
& \tau_{b s, s y s}^{s^{-}}=\tau_{b s}^{s a t}\left(1-\exp \left(-v \gamma^{s^{-}}\right)\right), \tau_{b s}^{s^{+}}=-A \tau_{b s}^{s^{-}} \text {if } \tau_{b s}^{s^{-}}>0
\end{aligned}
$$

where $\tau_{b s 0}^{s^{+}}$is the value of backstress at the point of the strain reversal and $\gamma_{b}$ is a fitting parameter. Similar relations were used in (Beyerlein and Tomé, 2007; Zecevic and Knezevic, 2015). When forming the backstress tensor, $\boldsymbol{\sigma}_{b s}^{c, f}$, parameter $\mathrm{A}$ is taken to be unity for the 
backstresses in $2 \sum_{s^{\prime}} \mathbf{m}^{c, s} \cdot \mathbf{m}^{c, s^{\prime}} \tau_{b s, s y s}^{s^{\prime}}$, since micro-backstresses are not long-range and do not contribute to the backstress on other slip systems. In other words, the micro-backstresses affect only the slip system on which they are generated and do not interact with other slip systems.

The backstress tensor defined with Eq. (30), $\dot{\boldsymbol{\sigma}}_{b s}^{c, f}$, will vary from grain to grain having a net volume average value of $\left\langle\dot{\boldsymbol{\sigma}}_{b s}^{c, f}\right\rangle$ (Brown and Stobbs, 1971).

The rate forms of Eqs. (29) and (30) are:

$$
\begin{aligned}
& \dot{\boldsymbol{\sigma}}_{b s}^{c, f}=2 \sum_{s}{ }^{\prime} \mathbf{m}^{c, s^{\prime}} \dot{\tau}_{b s, s y s}^{s^{\prime}}, \\
& \dot{\tau}_{b s}^{s}=\dot{\tau}_{b s, s y s}^{s}+2 \sum_{s^{\prime}} \mathbf{m}^{c, s} \cdot \mathbf{m}^{c, s^{\prime}} \dot{\tau}_{b s, s y s}^{s^{\prime}} \text { and } s^{\prime} \neq s,
\end{aligned}
$$

where the backstress rates can easily be evaluated using the chain rule: $\dot{\tau}_{b s, s y s}^{s}=\frac{\partial \tau_{b s, s y s}^{s}}{\partial \gamma^{s}} \dot{\gamma}^{s}$. The rates are needed to verify the loading condition: $\mathbf{m}^{c, s} \cdot \widehat{\boldsymbol{\sigma}}^{c}-\dot{\tau}_{b s}^{c, s}=\dot{\tau}_{c}^{c, s}$ as well as the derivative $\frac{\partial \tau_{b s, s y s}^{s}}{\partial \gamma^{s}}$ is needed for backstress matrix, $h_{b s}^{s s^{\prime}}$.

\subsubsection{Martensite grains}

In martensite regions the backstress rate is defined based on the volume average of backstress rate in ferrite phase, $\left\langle\dot{\boldsymbol{\sigma}}_{b s}^{c, f}\right\rangle$. From the condition that backstress in the sample vanishes, the rate of backstress in martensite regions is defined as:

$$
\left\langle\dot{\boldsymbol{\sigma}}_{b s}^{m r}\right\rangle=-\frac{V_{f}}{V_{m}}\left\langle\dot{\boldsymbol{\sigma}}_{b s}^{c, f}\right\rangle
$$

where $V_{f}$ and $V_{m}$ are phase fractions of ferrite and martensite phases respectively. The volume average of rate of backstress in martensite region is used to define the backstress on individual slip systems of martensite grains as:

$$
\dot{\tau}_{b s}^{c, s, m}=\mathbf{m}^{c, s} \cdot\left\langle\dot{\boldsymbol{\sigma}}_{b s}^{m r}\right\rangle .
$$

\section{Results}

In this section, the model is applied to simulate the cyclic tension-compression-tension response of DP 590 steel. We calibrate and critically validate the model against the experimental data presented in section 2 and provide insights into various aspects of the elasto-plastic material response during cyclic loading to large pre-strains.

The measured texture was represented using a set of 1000 weighted orientations representing the ferrite phase. To define crystal orientations of the martensite phase the following steps were performed: (1) a set of 100 random orientations are generated to represent prior austenite grains, which transform into martensite regions and (2) from 2 to 12 austenite-to-martensite variants out of 24 possible variants are randomly chosen to represent crystal orientations belonging to a martensite polycrystalline region. The relationship of martensite variants to the prior austenite grain followed the Kurdjumov-Sachs relationships (Morito et al., 2003). 
The layers around martensite regions are represented with randomly chosen ferrite crystal orientation. The thickness of the layer is adopted to be $\Delta r=0.25 r$, where $r$ is the radius of a spherical inclusion representing martensite region. For the material studied here, it is approximately $1 \mu \mathrm{m}$. Larger layer thickness of $\Delta r=0.822 r$ was used in (Taupin et al., 2013), where the thickness was adjusted to match the experimental data. In another study involving the dual phase steels (Kadkhodapour et al., 2011), it was found that the layer thickness is approximately $1.5 \mu \mathrm{m}$. However as denoted in (Cherkaoui et al., 1995), the solution procedure used is closely matching the exact solution for layer thicknesses up to $\Delta r=0.1 r$. The chosen value here represents the compromise between the physically measured value of the layer thickness and a value that will still give an accurate solution.

The deformation process is simulated by imposing strain increments in the RD direction, while ensuring that the overall normal stresses in ND and TD directions and the overall shear strains remain equal to zero.

\subsection{Identification of model parameters}

The parameters associated with the laws for evolution of slip system resistances and backstresses were identified using the cyclic tension-compression-tension curve deformed to a strain of 0.1 and slightly altered by comparing the cyclic curve deformed to a strain of 0.01 . The following slip system material parameters were identified for ferrite and martensite: initial slip resistance, $\tau_{0}^{\alpha}$, trapping rate coefficient, $k_{1}^{\alpha}$, activation barrier for de-pinning, $g^{\alpha}$, and drag stress, $D^{\alpha}$. In general, it is possible to obtain a reasonable estimate of these parameters from a single cyclic curve because each of these parameters predominantly affects a different portion of a stress-strain curve.

The initial slip resistance for martensite grains was approximated based on the carbon content in martensite as follows. The carbon content in DP 590 is between 0.04 and 0.15 wt. \% (Chung et al., 2010). Using the lever rule, the carbon content in martensite is evaluated to be between 0.21 and 1.53 wt. \%. An approximate relationship between yield stress, $\sigma_{y}$, for martensite and carbon content (C) in martensite is (Al-Abbasi and Nemes, 2007):

$$
\sigma_{y}=635+2687 \mathrm{C}[\mathrm{MPa}] \text {. }
$$

The value of slip resistance for martensite was estimated to be $880.5 \mathrm{MPa}$ by dividing the yield stress (Eq. 39) with the Taylor factor value (Stoller and Zinkle, 2000) and including the initial content of dislocation density as well as evaluating the accuracy of fits to the experimental data. The estimated slip resistance here is also in good agreement with values measured in other studies on DP600 and DP800 (Tasan et al., 2014a; Tasan et al., 2014b). It was noted that the initial slip resistance rapidly hardens, and it is this value that is approximately the same as the value we estimated here.

The volume expansion during phase transformation from austenite to martensite is causing the deformation of ferrite phase and accumulation of GNDs and statistically stored dislocations around the martensite regions (Kadkhodapour et al., 2011). The initial GND content in DP steel has been estimated based on the calculations of the kernel average misorientation and the 
dislocation tensor. It was found that the GND density decreases for an order of magnitude going from marteniste-ferrite interface to the interior of the ferrite grains (Calcagnotto et al., 2010). Therefore it is reasonable to assume that the content of initial dislocation density within the layer is an order of magnitude larger from that in ferrite grains. The increased GND content results in increase of yield stress of the region around marteniste islands. Finally, the kinematic backstress parameters for ferrite and layer are: saturation value for backstresses $\tau_{b s}^{s a t}$, asymmetry factor, A, and parameters $v$ and $\gamma_{b}$.

The established parameters are given in Table I and Table II for the hardening law and for the backstress law, respectively. Typical predictions of backstresses on slip systems in ferrite grains are shown in Fig. 7.

Table I. Model parameters for evolution of slip resistance of $\{110\}\langle 1 \overline{1} 1\rangle$ and $\{112\}\langle 11 \overline{1}\rangle$ slip modes.

\begin{tabular}{|l|l|l|l|l|l|}
\hline & $\tau_{0}^{\alpha}[\mathrm{MPa}]$ & $k_{1}^{\alpha}\left[\mathrm{m}^{-1}\right]$ & $g^{\alpha}$ & $D^{\alpha}[\mathrm{MPa}]$ & $q^{\alpha}$ \\
\hline Ferrite & 103 & $0.85 \times 10^{8}$ & 0.0013 & $1.326 \times 10^{4}$ & 40 \\
\hline Martensite & 830 & $0.3 \times 10^{8}$ & 0.0013 & $0.88 \times 10^{4}$ & 40 \\
\hline
\end{tabular}

Table II. Model parameters for evolution of backstresses in the ferrite grains and the layer.

\begin{tabular}{|l|l|l|l|l|}
\hline & $\tau_{b s}^{\text {sat }}[\mathrm{MPa}]$ & $v$ & $\gamma_{b}$ & $A$ \\
\hline Ferrite & 27 & 200 & 0.0015 & 4 \\
\hline
\end{tabular}

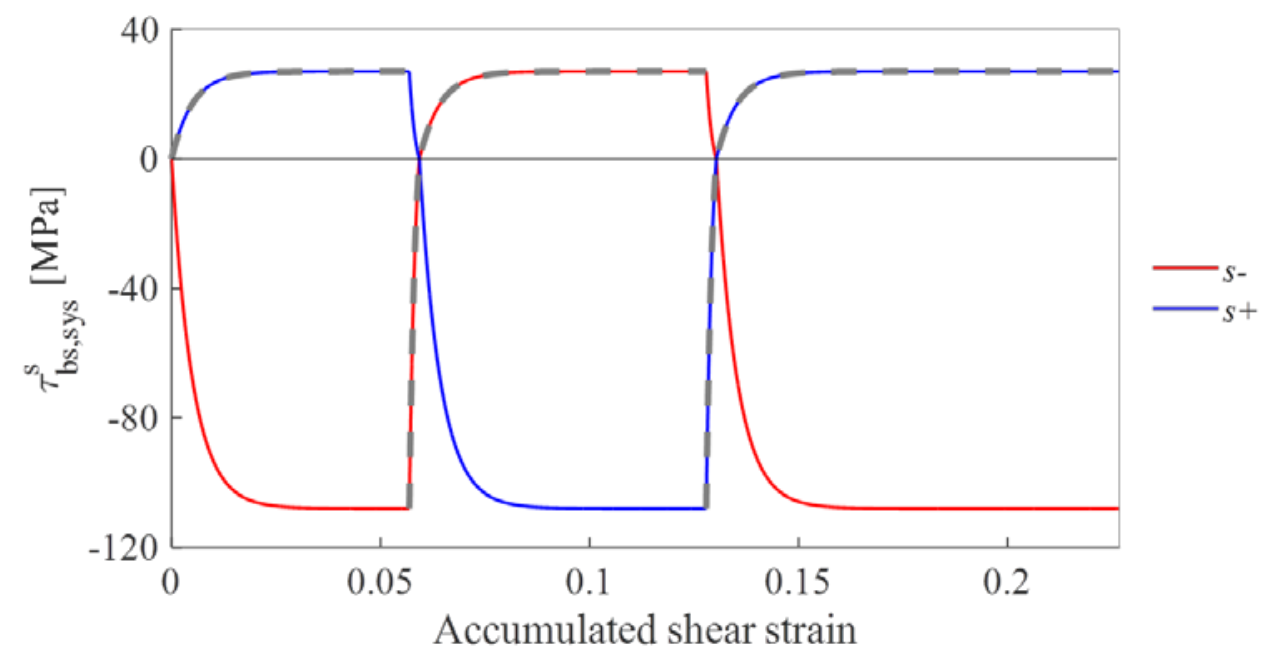

Fig. 7. Evolution of backstresses in a ferrite grain on the $s^{\text {th }}$ slip system as a function of accumulated shear strain during the forward tension and the $1^{\text {st }}$ and the $2^{\text {nd }}$ reversal. 


\subsection{Predictions of mechanical response}

Figure 8 shows the comparison between simulated and experimental cyclic tensioncompression-tension true stress-true strain curves to several levels of pre-strain. It can be seen that the model is capable of capturing all features particular to the cyclic behavior of DP 590: decreasing hardening rate during forward loading, non-linear unloading followed by the BE upon strain reversals, permanent softening and underlying changes in hardening rates during the $1^{\text {st }}$ and the $2^{\text {nd }}$ reverse loadings. A key achievement is that the model provides excellent agreement for all cyclic responses using a single set of parameters identified for DP 590 steel.
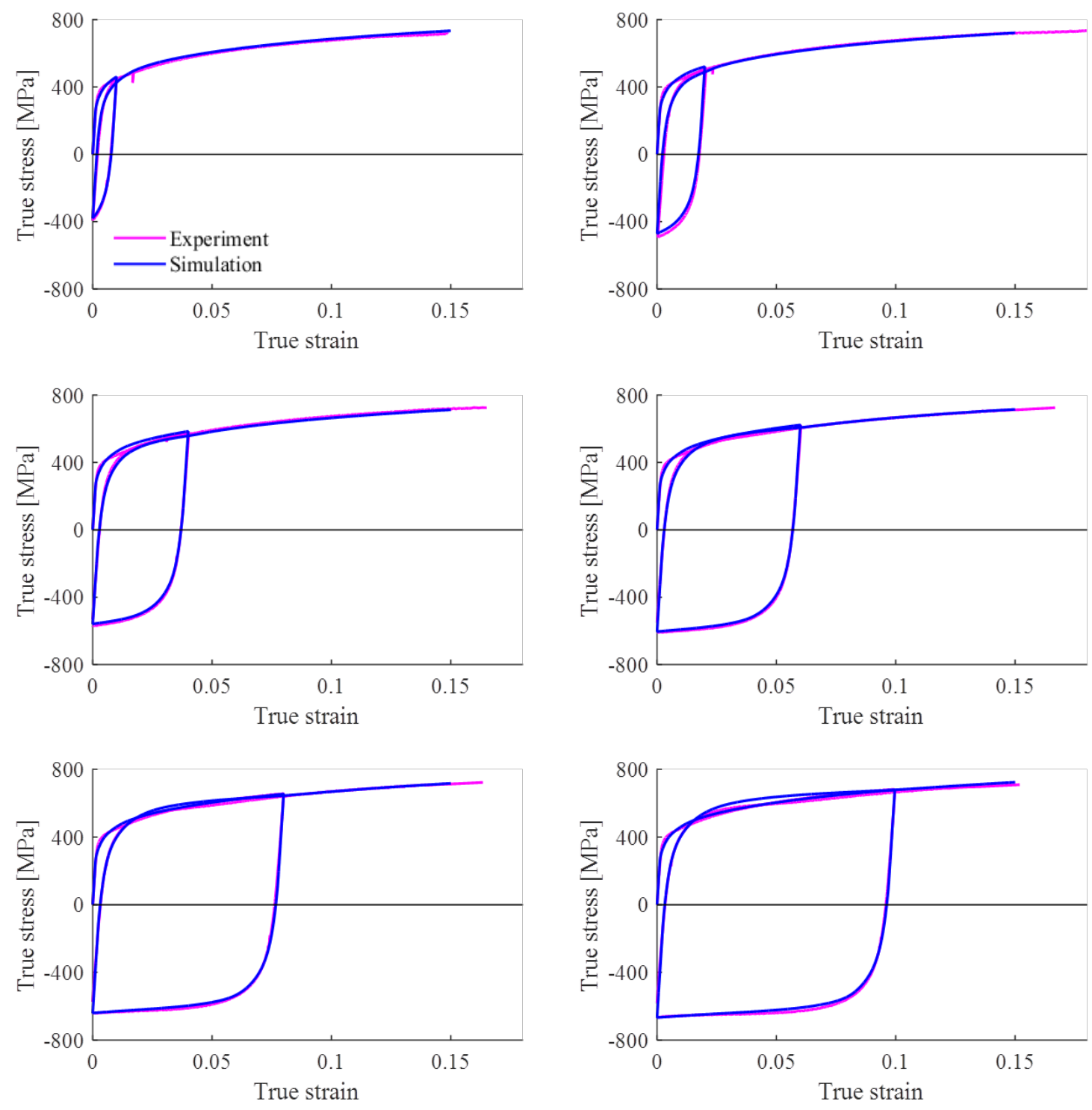

Fig. 8. Comparison of measured and simulated cyclic true stress-true strain curves for DP 590 steel. 


\section{Discussion}

Predictions of the cyclic mechanical behavior for DP 590 resulted from a combination of several distinct physical mechanisms incorporated in the model. In particular, these are: backstresses, dislocation annihilation, the layer representing localized hardening due to GNDs, and the two-level SC homogenizations. To isolate the individual contribution of these mechanisms on the material response during cyclic loading, we performed the simulations without (1) the $2^{\text {nd }}$ level homogenization, (2) the layer around martensite regions, (3) backstresses in martensite grains, and (4) dislocation annihilations. Additionally, we have performed simulations with the martensite phase being elastic. The effects are shown in Fig. 9.

To illustrate that incorporation of the additional physical mechanisms and the two-level homogenization scheme was essential and necessary for the prediction of the cyclic deformation of DP streel, we begin by showing the performance of the model presented in (Zecevic and Knezevic, 2015). Figure 9a and b show the predictions for two values of the initial slip resistance for martensite. It can be seen that the higher value of the initial slip resistance underpredicts the $\mathrm{BE}$ upon the first reversal and overpredicts the BE upon the second reversal, while the lower value of the initial slip resistance substantially overpredicts the BE upon the second reversal. Clearly the model originally developed for single-phase aluminum alloys (Zecevic and Knezevic, 2015) has benefited from the improvements described herein. 
a

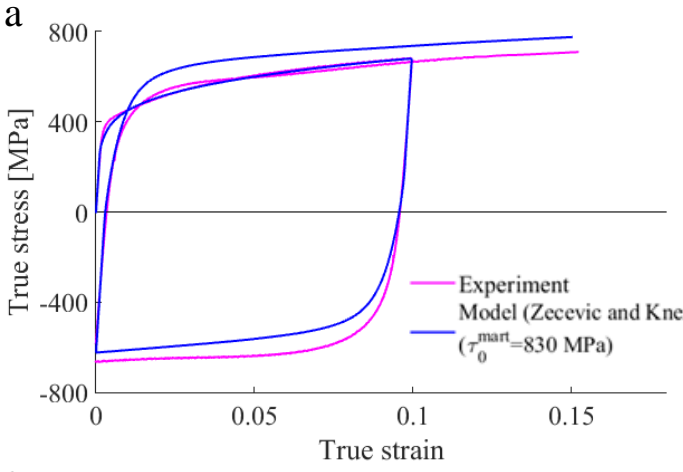

C

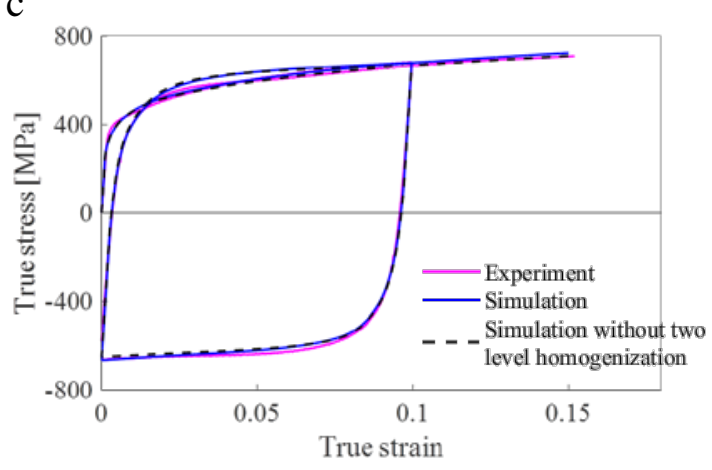

e
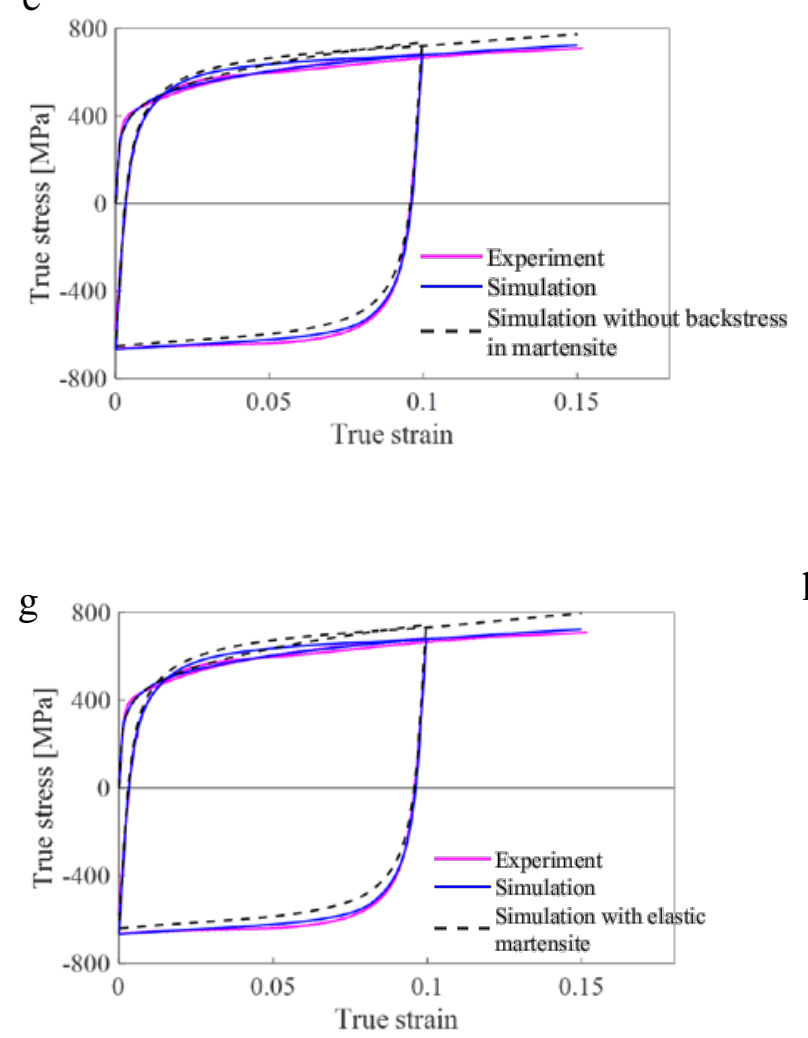

b

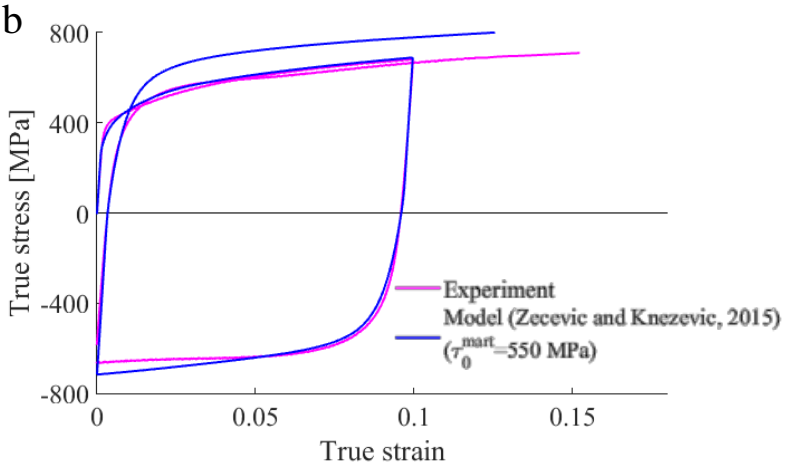

d

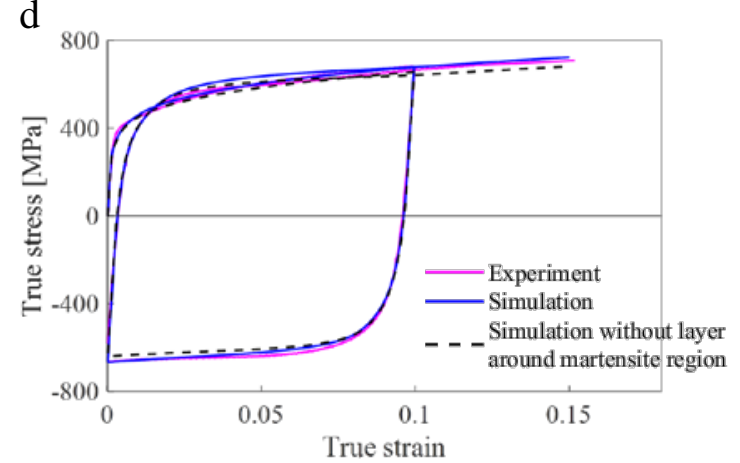

f

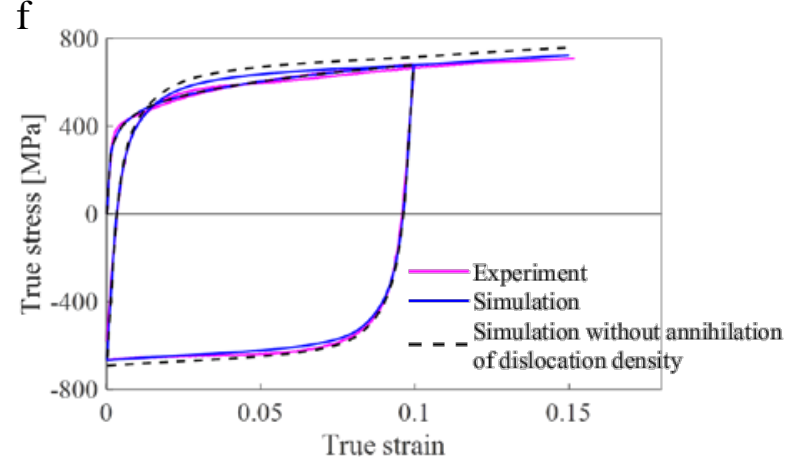

h

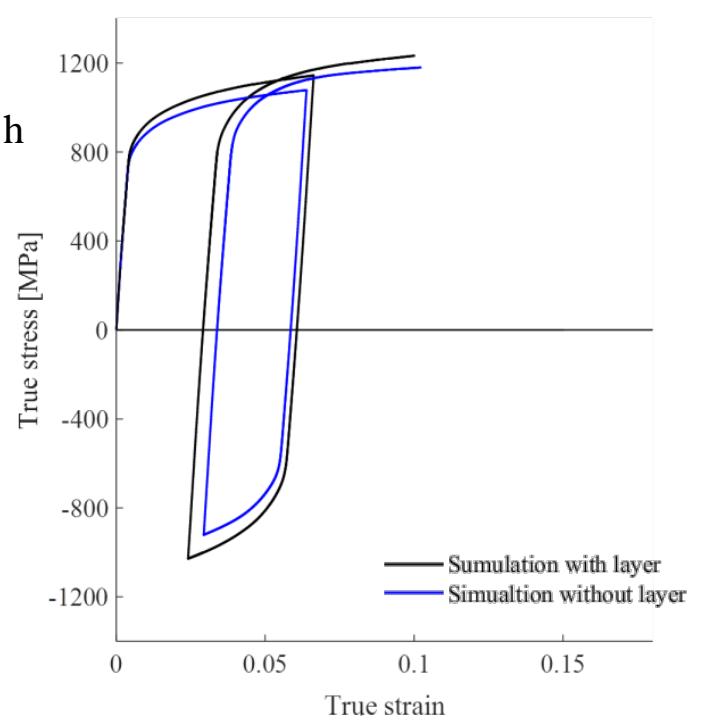


Fig. 9. Comparison of simulated and measured cyclic true stress-true strain response to a presstrain level of 0.1 using: the earlier version of EPSC for single-phase metals with an initial value of slip resistance for martensite phase of (a) $830 \mathrm{MPa}$ and (b) $550 \mathrm{MPa}$, and the new phase EPSC model (c) without $2^{\text {nd }}$ level homogenization for martensite regions, (d) without the layer around martensite regions, (e) without backstress in martensite grains, (f) without dislocation annihilations, (g) with elastic martensite. (h) Better illustrates the effect of the GND layer around martensite regions.

\subsection{Effect of two-level homogenization}

Figure 9c shows the experimental cyclic stress strain curve compared with the simulated stress strain curves deformed to a pre-strain level of 0.1 with and without the two-level homogenization. The effect of the $2^{\text {nd }}$ level homogenization for evaluation of properties of martensite regions does not appear to substantially influence the predictions. This could be associated to the fact that the DP 590 steel contains a relatively low volume fraction of martensite.

\subsection{Effect of the GND layer around the martensite regions}

The layer alters material properties in vicinity of the martensite regions. In order for martensite grains to yield in the early stage of deformation, a higher value of stress is necessary to be imposed over the martensite region. GNDs are believed to have a dominant role in elevating the stress in the martensite regions by altering material properties around the martensite regions and introducing backstresses. Comparison of the experimental and simulated cyclic curves pre-strained to 0.1 strain level with and without the GND layer around martensite is shown in Fig. 9d. Additionally, the effect of the layer is illustrated in a stress-strain plot solely for martensite phase in Fig. 9h. It can be seen that the effect of the layer reflects in the increase of the overall stress as well as the stress in the martensite region. These predictions are consistent with the results reported in (Taupin et al., 2013). The effect is dominant in the initial portion of deformation to approximately 0.02 strain level since in this portion of deformation the layer has substantially different properties than the HEM. The increased stiffness of the layer is the consequence of the localized hardening, which is due to the higher value of the initial dislocation content and backstresses evolving rapidly while the martensite phase is still elastic. Therefore the desired hardening effect in the early stage of deformation of the overall polycrystal can be achieved through the use of the reinforcing layer around the martensite region.

\subsection{Effect of backstresses}

The stress fields present in the material are the superposition of applied deformation (type I stresses), the intergranular stress field due to the interaction of individual single crystals with the matrix (type II stresses) and the backstress fields coming from GNDs around the martensite regions as well as the intra-granular dislocation structures (type III stresses). The backstress fields are evolved according to the algorithm outlined in Section 3.6. The total stress in each crystal is calculated as the sum of the interaction stress (sum of the applied stress and the 
intergranular interaction stress) and the backstress. Since the backstress fields directly enter the resolved shear stress calculations, they have an important role in determining the instantaneous elastic-plastic stiffness of individual grains. The backstress in ferrite will hinder the process of plastic deformation, since it will act against the applied field. However, since the volume average of the backstress field in the overall material has to be zero, the backstress in the martensite phase will be opposite to the one present in the ferrite phase and will act together with the applied field, aiding the driving force for plastic deformation. Having in mind that volume fraction of martensite is quite low in this material, the backstress in martensite will have a high value. The volume average backstresses in ferrite and martensite are shown in Figure 10. While martensite is elastic the condition of zero volume average of backstress in the overall polycrystal is clearly satisfied. However, when martensite becomes plastic additional evolution of backstress in ferrite has its source in heterogeneous dislocation distribution within individual ferrite grains (type III stress).

The absence of the backstresses in martensite would lead to a lower stress, which now would only be due to the interaction stress field and, in turn, often not sufficient to cause yielding in martensite. This would prolong elastic deformation of martensite causing an increase in magnitude of the grain interaction stresses in the martensite phase. In this case, for martensite to yield while obtaining good comparison with the experimental data, the initial slip resistance would need to take an unreasonable value of approximately $550 \mathrm{MPa}$. The increased grain interaction stress in martensite due to it deforming elastically is seen as the increase in the overall stress in Fig. 9e, where the effect of martensite backstresses on the 0.1 pre-strain cyclic curve prediction is shown. The increased grain interaction stresses in the martensite phase cause increase in intergranular residual stresses (the type II stresses), which reduce the permanent softening and negatively influence the BE predictions. Therefore the inclusion of the backstresses arising from local dislocation structures is essential for the predictions of accurate stress levels in the martensite regions having a reasonable value of slip resistance. Moreover, the results suggest that the internal backstresses have more pronounced effect on model predictions than the localized hardening from the GND layer. 


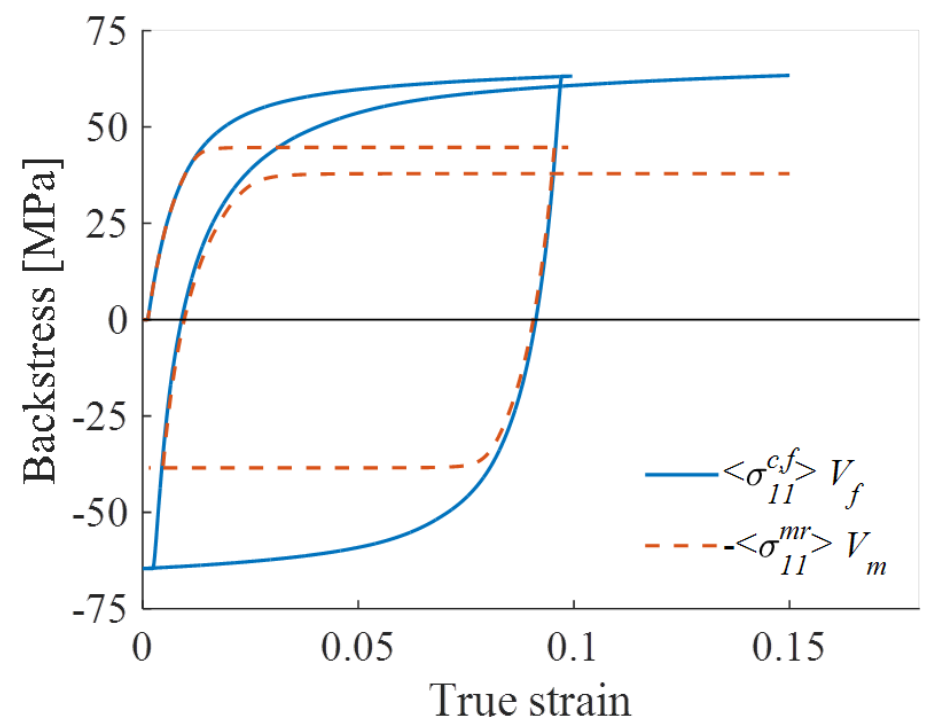

Fig. 10. Evolution of backstress in ferrite and martensite phase. It can be seed that the two backstresses are not in balance. The difference stems from the intra-granular sources (type III stresses) in ferrite.

\subsection{Effect of annihilation of dislocations}

Figure $9 \mathrm{f}$ shows the effect of annihilation of dislocations on the prediction of the 0.1 prestrained cyclic curves. Clearly, the annihilation of dislocation densities is an important mechanism for accurate predictions of stress levels, especially during the $2^{\text {nd }}$ reversal. The importance of the incorporation of reversible dislocation density in the prediction of cyclic deformation for single-phase materials was discussed in detail in (Zecevic and Knezevic, 2015). Since these effects translate here to two-phase materials, the reader is referred to this earlier work. Figure 11 shows the dislocation density evolution during the cyclic deformation process. The predicted flat regions show that after the strain reversal a certain amount of deformation can be accumulated without a marked increase in dislocation density. These results are in agreement with the constitutive assumption made to develop cyclic plasticity models in (Ohno, 1982; Ohno and Kachi, 1986). 


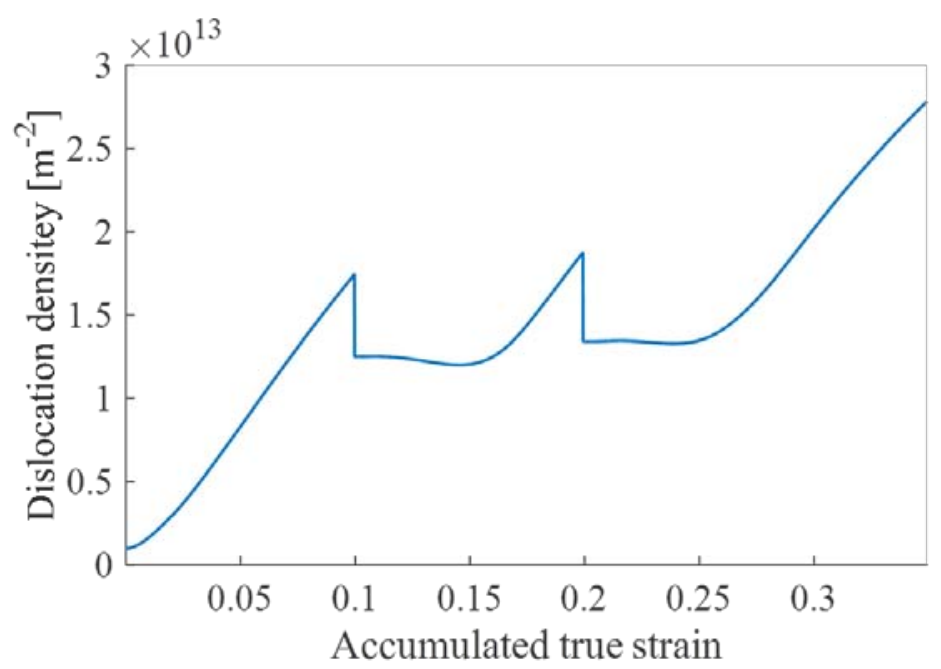

Fig. 11. Evolution of dislocation density for ferrite grains with dislocation annihilation.

\subsection{Effect of martensite deforming only elastically}

Most of the models used for simulating plastic deformation of DP steels reported in literature thus far consider the martensite phase to deform elastically (Böhlke, 2014; Yoshida et al., 2011). Figure $9 \mathrm{~g}$ depicts the effect of suppressing plastic deformation of the martensite phase. The increase in the overall hardening rate is a consequence of unrealistically large stresses in martensite grains and the accompanied intergranular residual stresses. While it would be possible to fit the forward tension experimental curve by adjusting the model parameters of the ferrite phase, this would lead to much worse predictions of the subsequent strain path reversal. In summary, the results suggest that accurate material models for DP steels must consider the martensite phase to deform plastically.

\section{Conclusions}

In this work, we developed a comprehensive polycrystal plasticity model capable of predicting the elasto-plastic cyclic deformation behavior of multi-phase polycrystalline metals deforming by crystallographic slip. To this end, we have extended the recently developed EPSC model for predicting cyclic deformation of single-phase metals (Zecevic and Knezevic, 2015) to include physics pertaining to the cyclic deformation of multi-phase materials, specifically dualphase (DP) steel. First, experimentally observed effects of GNDs around the martensite phase are accounted for by treating martensite regions as inclusions coated with a reinforcing layer. The layer facilitates modeling the localized hardening effects on the overall material response. Second, kinematic hardening at the slip system level through the evolution laws for backstresses for both ferrite and martensite is included. Third, dislocation annihilation mechanisms are included, to reflect the experimentally observed reduction of dislocation populations upon strain reversals. Fourth, two-level self-consistent homogenization has been implemented for calculations of properties of martensite polycrystalline regions. 
The performances of the developed model were evaluated on a data set collected for DP 590 steel sheets under in-plane cyclic tension-compression-tension deformation. The stress-strain response of the material exhibited the typical decreasing hardening rate in forward tensile deformation. The deformation upon the $1^{\text {st }}$ strain reversal started with a linear and then a nonlinear unloading, followed by the $\mathrm{BE}$, and continued under reduced hardening rate in comparison with that during forward loading. The deformation upon the $2^{\text {nd }}$ reversal exhibited similar characteristics to the $1^{\text {st }}$ reversal. It was shown that the model successfully captures the flow response of the material during cyclic tension-compression-tension deformation to various levels of plastic pre-strains. Specifically, hardening rates, non-linear unloading, and the BE were simultaneously captured using a single set of material parameters. From the favorable comparison of the predictions and experimental data, it is revealed that the backstresses predominately control the non-linear unloading and the $\mathrm{BE}$, and that the annihilation of dislocations plays the major role in capturing the $\mathrm{BE}$ and the changes in the hardening rates during reverse loading from those during forward loading. Consideration of the plastic deformation of the martensite phase aided by the GND layer was essential for accurate prediction of stress levels in that phase, as well as the overall material response of DP 590 steel. As these effects have major implications on the accuracy of numerical simulations of forming processes of DP steels, the good predictions by the present model represent a significant incentive for incorporating the model into finite-element simulation tools to facilitate treatment of complex, non-monotonic deformation processes with heterogeneous boundary conditions.

\section{Acknowledgements}

This work is based upon a project supported by the National Science Foundation under grant CMMI-1301081. The authors gratefully acknowledge this support. The EBSD work was performed in the University Instrumentation Center (UIC) at the University of New Hampshire (UNH). The authors wish to acknowledge help from Nancy Cherim and Mark A. Townley for assistance with operating the microscope at UIC. The steel sheets were provided by Dr. Ming Shi of US Steel. The authors also acknowledge numerous discussions with Professor Brad L. Kinsey of UNH.

\section{References}

Abel, A., 1987. Historical perspectives and some of the main features of the Bauschinger effect, Materials Forum. Institute of Metals and Materials Australasia, pp. 11-26.

Al-Abbasi, F.M., Nemes, J.A., 2007. Characterizing DP-steels using micromechanical modeling of cells. Computational Materials Science 39, 402-415.

Al-Harbi, H.F., Knezevic, M., Kalidindi, S.R., 2010. Spectral approaches for the fast computation of yield surfaces and first-order plastic property closures for polycrystalline materials with cubic-triclinic textures. CMC: Computers, Materials, \& Continua 15, 153-172.

Ardeljan, M., Beyerlein, I.J., Knezevic, M., 2014a. A dislocation density based crystal plasticity finite element model: Application to a two-phase polycrystalline HCP/BCC composites. Journal of the Mechanics and Physics of Solids 66, 16-31. 
Ardeljan, M., Knezevic, M., Nizolek, T., Beyerlein, I.J., Mara, N.A., Pollock, T.M., 2015a. A study of microstructure-driven strain localizations in two-phase polycrystalline HCP/BCC composites using a multi-scale model. International Journal of Plasticity 74, 35-57.

Ardeljan, M., Knezevic, M., Nizolek, T., Beyerlein, I.J., Zheng, S.J., Carpenter, J.S., McCabe, R.J., Mara, N.A., Pollock, T.M., 2014b. A multi-scale model for texture development in $\mathrm{Zr} / \mathrm{Nb}$ nanolayered composites processed by accumulative roll bonding. IOP Conference Series: Materials Science and Engineering 63, 012170.

Ardeljan, M., McCabe, R.J., Beyerlein, I.J., Knezevic, M., 2015b. Explicit incorporation of deformation twins into crystal plasticity finite element models. Computer Methods in Applied Mechanics and Engineering 295, 396-413.

Armstrong, P.J., Frederick, C.O., 1966. A mathematical representation of multiaxial Bauschinger effect. Berkeley nuclear laboratories.

Bate, P.S., Wilson, D.V., 1986. Analysis of the bauschinger effect. Acta Metallurgica 34, 10971105.

Bathe, K.-J., 1996. Finite element procedures. Englewood Cliffs, N.J.: Prentice Hall.

Bauschinger, J., 1886. Über die Veränderung der Elasticitätsgrenze und Festigkeit des Eisen und Stahls durch Strecken und Quetschen, durch Erwarmen und Abkühlen und durch oftmal wiederholte Beanspruchung. Mitteilungen aus dem mechanisch-technischen Laboratorium der k. polytechnischen Schule, 1877-1836.

Bayley, C.J., Brekelmans, W.A.M., Geers, M.G.D., 2006. A comparison of dislocation induced back stress formulations in strain gradient crystal plasticity. International Journal of Solids and Structures 43, 7268-7286.

Berbenni, S., Cherkaoui, M., 2010. Homogenization of multicoated inclusion-reinforced linear elastic composites with eigenstrains: Application to thermoelastic behavior. Philosophical Magazine 90, 3003-3026.

Beyerlein, I.J., 2008. Plastic Behavior of Metals in Reverse Straining after Large Pre-Strains. Materials Science Forum 579, 41-60.

Beyerlein, I.J., McCabe, R.J., Tomé, C.N., 2011. Effect of microstructure on the nucleation of deformation twins in polycrystalline high-purity magnesium: A multi-scale modeling study. Journal of the Mechanics and Physics of Solids 59, 988-1003.

Beyerlein, I.J., Tomé, C.N., 2007. Modeling transients in the mechanical response of copper due to strain path changes. International Journal of Plasticity 23, 640-664.

Beyerlein, I.J., Tomé, C.N., 2008. A dislocation-based constitutive law for pure Zr including temperature effects. International Journal of Plasticity 24, 867-895.

Bhattacharyya, A., Knezevic, M., Abouaf, M., 2015. Characterization of Crystallographic Texture and Intra-Grain Morphology in Cross-Rolled Tantalum. Metallurgical and Materials Transactions A 46, 1085-1096.

Böhlke, F.R.T., 2014. Microstructure based prediction and homogenization of the strain hardening behavior of dual-phase steel. Archive of Applied Mechanics 85, 1433-1458.

Brown, D.W., Beyerlein, I.J., Sisneros, T.A., Clausen, B., Tomé, C.N., 2012. Role of twinning and slip during compressive deformation of beryllium as a function of strain rate. International Journal of Plasticity 29, 120-135.

Brown, L.M., Stobbs, W.M., 1971. The Work-Hardening of Copper-Silica: I. A Model Based on Internal Stresses, With No Plastic Relaxation. Philosophical Magazine 23, 1185-1199.

Cai, S., Daymond, M.R., Holt, R.A., 2009. Modeling the room temperature deformation of a two-phase zirconium alloy. Acta Materialia 57, 407-419. 
Calcagnotto, M., Ponge, D., Demir, E., Raabe, D., 2010. Orientation gradients and geometrically necessary dislocations in ultrafine grained dual-phase steels studied by 2D and 3D EBSD. Materials Science and Engineering: A 527, 2738-2746.

Chaboche, J., Rousselier, G., 1983. On the plastic and viscoplastic constitutive equations-Part I: Rules developed with internal variable concept. Journal of Pressure Vessel Technology 105, 153-158.

Chaboche, J.L., 1977. Viscoplastic constitutive equations for the description of cyclic and ansiotropic behavior of metals, In: XVIIth Polish solid mechanics conference, Bulletin de l’Académie Polonaise des Sciences, Série Sciences et Techniques, pp. 33-41.

Chaboche, J.L., 2008. A review of some plasticity and viscoplasticity constitutive theories. International Journal of Plasticity 24, 1642-1693.

Cherkaoui, M., Sabar, H., Berveiller, M., 1995. Elastic composites with coated reinforcements: A micromechanical approach for nonhomothetic topology. International Journal of Engineering Science 33, 829-843.

Choi, S.H., Kim, E.Y., Woo, W., Han, S.H., Kwak, J.H., 2013. The effect of crystallographic orientation on the micromechanical deformation and failure behaviors of DP980 steel during uniaxial tension. International Journal of Plasticity 45, 85-102.

Chung, K., Lee, W., Kim, D., Kim, J., Chung, K.-H., Kim, C., Okamoto, K., Wagoner, R.H., 2010. Macro-performance evaluation of friction stir welded automotive tailor-welded blank sheets: Part I - Material properties. International Journal of Solids and Structures 47, 10481062.

Cullen, G.W., Korkolis, Y.P., 2013. Ductility of 304 stainless steel under pulsed uniaxial loading. International Journal of Solids and Structures 50, 1621-1633.

Demir, E., Raabe, D., 2010. Mechanical and microstructural single-crystal Bauschinger effects: Observation of reversible plasticity in copper during bending. Acta Materialia 58, 6055-6063.

Deng, N., Kuwabara, T., Korkolis, Y.P., 2015. On the non-linear unloading behavior of a biaxially loaded dual-phase steel sheet. (in preparation).

Eshelby, J.D., 1957. The determination of the elastic field of an ellipsoidal inclusion, and related problems. Proc R. Soc. Lond. A 241, 376-396.

Evers, L.P., Brekelmans, W.A.M., Geers, M.G.D., 2004. Non-local crystal plasticity model with intrinsic SSD and GND effects. Journal of the Mechanics and Physics of Solids 52, 23792401.

Fast, T., Knezevic, M., Kalidindi, S.R., 2008. Application of microstructure sensitive design to structural components produced from hexagonal polycrystalline metals. Computational Materials Science 43, 374-383.

Fleck, N.A., Muller, G.M., Ashby, M.F., Hutchinson, J.W., 1994. Strain gradient plasticity: Theory and experiment. Acta Metallurgica et Materialia 42, 475-487.

Gardey, B., Bouvier, S., Bacroix, B., 2005. Correlation between the macroscopic behavior and the microstructural evolutions during large plastic deformation of a dual-phase steel. Metallurgical and Materials Transactions A 36, 2937-2945.

Goh, C.-H., Neu, R.W., McDowell, D.L., 2003. Crystallographic plasticity in fretting of Ti6AL-4V. International Journal of Plasticity 19, 1627-1650.

Gong, H., Wang, S., Knysh, P., Korkolis, Y.P., 2016. Experimental investigation of the mechanical response of laser-welded dissimilar blanks from advanced- and ultra-highstrength steels. Materials \& Design 90, 1115-1123. 
Gough, H., Hanson, D., Wright, S., 1927. The Behaviour of Single Crystals of Aluminium under Static and Repeated Stresses. Philosophical Transactions of the Royal Society of London. Series A, Containing Papers of a Mathematical or Physical Character, 1-30.

Gracio, J.J., Barlat, F., Rauch, E.F., Jones, P.T., Neto, V.F., Lopes, A.B., 2004. Artificial aging and shear deformation behaviour of 6022 aluminium alloy. International Journal of Plasticity 20, 427-445.

Harder, J., 1999. A crystallographic model for the study of local deformation processes in polycrystals. International Journal of Plasticity 15, 605-624.

Hasegawa, T., Yakou, T., Karashima, S., 1975. Deformation behaviour and dislocation structures upon stress reversal in polycrystalline aluminium. Materials Science and Engineering 20, 267-276.

Hosford, W.F., Caddell, R.M., 1993. Metal forming mechanics and metallurgy. Prentice-Hall, Inc.

Hu, Z., Rauch, E.F., Teodosiu, C., 1992. Work-hardening behavior of mild steel under stress reversal at large strains. International Journal of Plasticity 8, 839-856.

Jahedi, M., Knezevic, M., Paydar, M., 2015a. High-Pressure Double Torsion as a Severe Plastic Deformation Process: Experimental Procedure and Finite Element Modeling. J. of Materi Eng and Perform 24, 1471-1482.

Jahedi, M., Paydar, M.H., Knezevic, M., 2015b. Enhanced microstructural homogeneity in metal-matrix composites developed under high-pressure-double-torsion. Materials Characterization 104, 92-100.

Jahedi, M., Paydar, M.H., Zheng, S., Beyerlein, I.J., Knezevic, M., 2014. Texture evolution and enhanced grain refinement under high-pressure-double-torsion. Materials Science and Engineering: A 611, 29-36.

Kadkhodapour, J., Schmauder, S., Raabe, D., Ziaei-Rad, S., Weber, U., Calcagnotto, M., 2011. Experimental and numerical study on geometrically necessary dislocations and nonhomogeneous mechanical properties of the ferrite phase in dual phase steels. Acta Materialia 59, 4387-4394.

Kalidindi, S.R., Bronkhorst, C.A., Anand, L., 1992. Crystallographic texture evolution in bulk deformation processing of FCC metals. Journal of the Mechanics and Physics of Solids 40, 537-569.

Kalidindi, S.R., Duvvuru, H.K., Knezevic, M., 2006. Spectral calibration of crystal plasticity models. Acta Materialia 54, 1795-1804.

Kassner, M.E., Geantil, P., Levine, L.E., 2013. Long range internal stresses in single-phase crystalline materials. International Journal of Plasticity 45, 44-60.

Kim, J.H., Kim, D., Barlat, F., Lee, M.-G., 2012. Crystal plasticity approach for predicting the Bauschinger effect in dual-phase steels. Materials Science and Engineering: A 539, 259-270.

Kitayama, K., Tomé, C.N., Rauch, E.F., Gracio, J.J., Barlat, F., 2013. A crystallographic dislocation model for describing hardening of polycrystals during strain path changes. Application to low carbon steels. International Journal of Plasticity in press.

Knezevic, M., Al-Harbi, H.F., Kalidindi, S.R., 2009. Crystal plasticity simulations using discrete Fourier transforms. Acta Materialia 57, 1777-1784.

Knezevic, M., Beyerlein, I.J., Brown, D.W., Sisneros, T.A., Tomé, C.N., 2013a. A polycrystal plasticity model for predicting mechanical response and texture evolution during strain-path changes: Application to beryllium. International Journal of Plasticity 49, 185-198. 
Knezevic, M., Beyerlein, I.J., Lovato, M.L., Tomé, C.N., Richards, A.W., McCabe, R.J., 2014a. A strain-rate and temperature dependent constitutive model for BCC metals incorporating non-Schmid effects: Application to tantalum-tungsten alloys. International Journal of Plasticity 62, 93-104.

Knezevic, M., Beyerlein, I.J., Nizolek, T., Mara, N.A., Pollock, T.M., 2013b. Anomalous Basal Slip Activity in Zirconium under High-strain Deformation. Materials Research Letters 1, 133-140.

Knezevic, M., Capolungo, L., Tomé, C.N., Lebensohn, R.A., Alexander, D.J., Mihaila, B., McCabe, R.J., 2012. Anisotropic stress-strain response and microstructure evolution of textured $\alpha$-uranium. Acta Materialia 60, 702-715.

Knezevic, M., Carpenter, J.S., Lovato, M.L., McCabe, R.J., 2014b. Deformation behavior of the cobalt-based superalloy Haynes 25: Experimental characterization and crystal plasticity modeling. Acta Materialia 63, 162-168.

Knezevic, M., Drach, B., Ardeljan, M., Beyerlein, I.J., 2014c. Three dimensional predictions of grain scale plasticity and grain boundaries using crystal plasticity finite element models. Computer Methods in Applied Mechanics and Engineering 277, 239-259.

Knezevic, M., Jahedi, M., Korkolis, Y.P., Beyerlein, I.J., 2014d. Material-based design of the extrusion of bimetallic tubes. Computational Materials Science 95, 63-73.

Knezevic, M., Kalidindi, S.R., 2007. Fast computation of first-order elastic-plastic closures for polycrystalline cubic-orthorhombic microstructures. Computational Materials Science 39, 643-648.

Knezevic, M., Kalidindi, S.R., Fullwood, D., 2008. Computationally efficient database and spectral interpolation for fully plastic Taylor-type crystal plasticity calculations of facecentered cubic polycrystals. International Journal of Plasticity 24, 1264-1276.

Knezevic, M., Landry, N.W., 2015. Procedures for reducing large datasets of crystal orientations using generalized spherical harmonics. Mechanics of Materials 88, 73-86.

Knezevic, M., Lebensohn, R.A., Cazacu, O., Revil-Baudard, B., Proust, G., Vogel, S.C., Nixon, M.E., 2013c. Modeling bending of $\alpha$-titanium with embedded polycrystal plasticity in implicit finite elements. Materials Science and Engineering: A 564, 116-126.

Knezevic, M., Levinson, A., Harris, R., Mishra, R.K., Doherty, R.D., Kalidindi, S.R., 2010. Deformation twinning in AZ31: Influence on strain hardening and texture evolution. Acta Materialia 58, 6230-6242.

Knezevic, M., McCabe, R.J., Lebensohn, R.A., Tomé, C.N., Liu, C., Lovato, M.L., Mihaila, B., 2013d. Integration of self-consistent polycrystal plasticity with dislocation density based hardening laws within an implicit finite element framework: Application to low-symmetry metals. Journal of the Mechanics and Physics of Solids 61, 2034-2046.

Knezevic, M., McCabe, R.J., Tomé, C.N., Lebensohn, R.A., Chen, S.R., Cady, C.M., Gray Iii, G.T., Mihaila, B., 2013e. Modeling mechanical response and texture evolution of $\alpha$-uranium as a function of strain rate and temperature using polycrystal plasticity. International Journal of Plasticity 43, 70-84.

Knezevic, M., Nizolek, T., Ardeljan, M., Beyerlein, I.J., Mara, N.A., Pollock, T.M., 2014e. Texture evolution in two-phase $\mathrm{Zr} / \mathrm{Nb}$ lamellar composites during accumulative roll bonding. International Journal of Plasticity 57, 16-28.

Knezevic, M., Savage, D.J., 2014. A high-performance computational framework for fast crystal plasticity simulations. Computational Materials Science 83, 101-106. 
Knezevic, M., Zecevic, M., Beyerlein, I.J., Bingert, J.F., McCabe, R.J., 2015. Strain rate and temperature effects on the selection of primary and secondary slip and twinning systems in HCP Zr. Acta Materialia 88, 55-73.

Kocks, U.F., Mecking, H., 1981. Kinetics of Flow and Strain-Hardening. Acta Metallurgica 29, 1865-1875.

Korzekwa, D.A., Matlock, D.K., Krauss, G., 1984. Dislocation substructure as a function of strain in a dual-phase steel. MTA 15, 1221-1228.

Kuwabara, T., Kumano, Y., Ziegelheim, J., Kurosaki, I., 2009. Tension-compression asymmetry of phosphor bronze for electronic parts and its effect on bending behavior. International Journal of Plasticity 25, 1759-1776.

Kuwabara, T., Nagata, K., Nakako, T., 2001. Measurement and analysis of the Bauschinger effect of sheet metals subjected to in plane stress reversals. Proceedings of AMPT 1, 407412.

Lebensohn, R.A., Tomé, C.N., 1993. A self-consistent anisotropic approach for the simulation of plastic deformation and texture development of polycrystals: Application to zirconium alloys. Acta Metallurgica et Materialia 41, 2611-2624.

Lentz, M., Klaus, M., Beyerlein, I.J., Zecevic, M., Reimers, W., Knezevic, M., 2015a. In situ Xray diffraction and crystal plasticity modeling of the deformation behavior of extruded MgLi-(Al) alloys: An uncommon tension-compression asymmetry. Acta Materialia 86, 254268.

Lentz, M., Klaus, M., Wagner, M., Fahrenson, C., Beyerlein, I.J., Zecevic, M., Reimers, W., Knezevic, M., 2015b. Effect of age hardening on the deformation behavior of an Mg-Y-Nd alloy: In-situ X-ray diffraction and crystal plasticity modeling. Materials Science and Engineering: A 628, 396-409.

Li, K., Carden, W., Wagoner, R., 2002. Simulation of springback. International Journal of Mechanical Sciences 44, 103-122.

Li, L., Shen, L., Proust, G., 2014. A texture-based representative volume element crystal plasticity model for predicting Bauschinger effect during cyclic loading. Materials Science and Engineering: A 608, 174-183.

Lipinski, P., Berveiller, M., 1989. Elastoplasticity of micro-inhomogeneous metals at large strains. International Journal of Plasticity 5, 149-172.

Ma, A., Roters, F., Raabe, D., 2006a. A dislocation density based constitutive model for crystal plasticity FEM including geometrically necessary dislocations. Acta Materialia 54, 21692179.

Ma, A., Roters, F., Raabe, D., 2006b. On the consideration of interactions between dislocations and grain boundaries in crystal plasticity finite element modeling - Theory, experiments, and simulations. Acta Materialia 54, 2181-2194.

Ma, A., Roters, F., Raabe, D., 2007. A dislocation density based constitutive law for BCC materials in crystal plasticity FEM. Computational Materials Science 39, 91-95.

Madec, R., Devincre, B., Kubin, L., Hoc, T., Rodney, D., 2003. The role of collinear interaction in dislocation-induced hardening. Science 301, 1879-1882.

McDowell, D.L., 1992. A nonlinear kinematic hardening theory for cyclic thermoplasticity and thermoviscoplasticity. International Journal of Plasticity 8, 695-728.

Meyers, M.A., Chawla, K.K., 2009. Mechanical behavior of materials, Cambridge. 
Mompiou, F., Caillard, D., Legros, M., Mughrabi, H., 2012. In situ TEM observations of reverse dislocation motion upon unloading in tensile-deformed UFG aluminium. Acta Materialia 60, 3402-3414.

Morito, S., Tanaka, H., Konishi, R., Furuhara, T., Maki, T., 2003. The morphology and crystallography of lath martensite in Fe-C alloys. Acta Materialia 51, 1789-1799.

Mughrabi, H., 1983. Dislocation wall and cell structures and long-range internal stresses in deformed metal crystals. Acta Metallurgica 31, 1367-1379.

Mughrabi, H., 2001. On the role of strain gradients and long-range internal stresses in the composite model of crystal plasticity. Materials Science and Engineering: A 317, 171-180.

Neil, C.J., Wollmershauser, J.A., Clausen, B., Tomé, C.N., Agnew, S.R., 2010. Modeling lattice strain evolution at finite strains and experimental verification for copper and stainless steel using in situ neutron diffraction. International Journal of Plasticity 26, 1772-1791.

Nesterova, E.V., Bouvier, S., Bacroix, B., 2015. Microstructure evolution and mechanical behavior of a high strength dual-phase steel under monotonic loading. Materials Characterization 100, 152-162.

Nieh, T.G., Nix, W.D., 1986. Unloading yield effects in aluminum alloys. Metallurgical transactions. A, Physical metallurgy and materials science 17 A, 121-126.

Ohno, N., 1982. A Constitutive Model of Cyclic Plasticity With a Nonhardening Strain Region. J. Appl. Mech. 49(4), 721-727

Ohno, N., Kachi, Y., 1986. A Constitutive Model of Cyclic Plasticity for Nonlinear Hardening Materials. J. Appl. Mech. 53(2), 395-403.

Orowan, E., 1959. Causes and Effects of Internal Stresses, General Motors Symposium, ed. G.M. Rassweiler and W.L. Grube. Elsevier, Amsterdam, pp. 59-80.

Pavlina, E., Lee, M.-G., Barlat, F., 2015. Observations on the Nonlinear Unloading Behavior of Advanced High Strength Steels. Metallurgical and Materials Transactions A 46, 18-22.

Pinard, P.T., Schwedt, A., Ramazani, A., Prahl, U., Richter, S., 2013. Characterization of DualPhase Steel Microstructure by Combined Submicrometer EBSD and EPMA Carbon Measurements. Microscopy and Microanalysis 19, 996-1006.

Roters, F., Eisenlohr, P., Hantcherli, L., Tjahjanto, D.D., Bieler, T.R., Raabe, D., 2010. Overview of constitutive laws, kinematics, homogenization and multiscale methods in crystal plasticity finite-element modeling: Theory, experiments, applications. Acta Materialia 58, 1152-1211.

Sachs, G., 1929. Zur ableitung einer fliessbedingung, Mitteilungen der deutschen Materialprüfungsanstalten. Springer, pp. 94-97.

Savage, D.J., Knezevic, M., 2015. Computer implementations of iterative and non-iterative crystal plasticity solvers on high performance graphics hardware. Computational Mechanics 56, 677-690.

Schwindt, C., Schlosser, F., Bertinetti, M.A., Stout, M., Signorelli, J.W., 2015. Experimental and Visco-Plastic Self-Consistent evaluation of forming limit diagrams for anisotropic sheet metals: An efficient and robust implementation of the M-K model. International Journal of Plasticity 73, 62-99.

Simmons, G., Wang, H., 1971. Single Crystal Elastic Constants and Calculated Aggregate Properties. MIT Press, Cambridge.

Smith, A., Chen, Z., Lee, J.Y., Lee, M.G., Wagoner, R.H., 2014. Effective method for fitting complex constitutive equations. International Journal of Plasticity 58, 100-119. 
Sritharan, T., Chandel, R.S., 1997. Phenomena in interrupted tensile tests of heat treated aluminium alloy 6061. Acta Materialia 45, 3155-3161.

Stoller, R.E., Zinkle, S.J., 2000. On the relationship between uniaxial yield strength and resolved shear stress in polycrystalline materials. Journal of Nuclear Materials 283-287, Part 1, 349352.

Stout, M., Rollett, A., 1990. Large-strain Bauschinger effects in fcc metals and alloys. Metallurgical and Materials Transactions A 21, 3201-3213.

Tasan, C.C., Diehl, M., Yan, D., Zambaldi, C., Shanthraj, P., Roters, F., Raabe, D., $2014 a$. Integrated experimental-simulation analysis of stress and strain partitioning in multiphase alloys. Acta Materialia 81, 386-400.

Tasan, C.C., Hoefnagels, J.P.M., Diehl, M., Yan, D., Roters, F., Raabe, D., 2014b. Strain localization and damage in dual phase steels investigated by coupled in-situ deformation experiments and crystal plasticity simulations. International Journal of Plasticity 63, 198-210.

Taupin, V., Berbenni, S., Fressengeas, C., Bouaziz, O., 2010. On particle size effects: An internal length mean field approach using field dislocation mechanics. Acta Materialia 58, 5532-5544.

Taupin, V., Pesci, R., Berbenni, S., Berveiller, S., Ouahab, R., Bouaziz, O., 2013. Lattice strain measurements using synchrotron diffraction to calibrate a micromechanical modeling in a ferrite-cementite steel. Materials Science and Engineering: A 561, 67-77.

Taylor, G.I., 1938. Plastic strain in metals. Journal of the Institute of Metals 62, 307-324.

Turner, P.A., Tomé, C.N., 1994. A study of residual stresses in Zircaloy-2 with rod texture. Acta Metallurgica et Materialia 42, 4143-4153.

Verma, R.K., Kuwabara, T., Chung, K., Haldar, A., 2011. Experimental evaluation and constitutive modeling of non-proportional deformation for asymmetric steels. International Journal of Plasticity 27, 82-101.

Wagoner, R.H., Lim, H., Lee, M.-G., 2013. Advanced Issues in springback. International Journal of Plasticity 45, 3-20.

Wen, W., Borodachenkova, M., Tomé, C.N., Vincze, G., Rauch, E.F., Barlat, F., Grácio, J.J., 2014. Mechanical behavior of $\mathrm{Mg}$ subjected to strain path changes: Experiments and modeling. International Journal of Plasticity.

Wilson, D.V., Bate, P.S., 1986. Reversibility in the work hardening of spheroidised steels. Acta Metallurgica 34, 1107-1120.

Wilson, D.V., Zandrahimi, M., Roberts, W.T., 1990. Effects of changes in strain path on workhardening in CP aluminium and an Al-Cu-Mg alloy. Acta Metallurgica et Materialia 38, 215226.

Withers, P.J., Bhadeshia, H.K.D.H., 2001. Residual stress. Part 1 - Measurement techniques. Materials Science and Technology 17, 355-365.

Wollmershauser, J.A., Clausen, B., Agnew, S.R., 2012. A slip system-based kinematic hardening model application to in situ neutron diffraction of cyclic deformation of austenitic stainless steel. International Journal of Fatigue 36, 181-193.

$\mathrm{Xu}$, B., Jiang, Y., 2004. A cyclic plasticity model for single crystals. International Journal of Plasticity 20, 2161-2178.

Yoshida, F., Uemori, T., Fujiwara, K., 2002. Elastic-plastic behavior of steel sheets under inplane cyclic tension-compression at large strain. International Journal of Plasticity 18, 633659. 
Yoshida, K., Brenner, R., Bacroix, B., Bouvier, S., 2011. Micromechanical modeling of the work-hardening behavior of single- and dual-phase steels under two-stage loading paths. Materials Science and Engineering: A 528, 1037-1046.

Zang, S.-l., Lee, M.-g., Hoon Kim, J., 2013. Evaluating the significance of hardening behavior and unloading modulus under strain reversal in sheet springback prediction. International Journal of Mechanical Sciences 77, 194-204.

Zecevic, M., Knezevic, M., 2015. A dislocation density based elasto-plastic self-consistent model for the prediction of cyclic deformation: Application to Al6022-T4. International Journal of Plasticity 72, 200-217.

Zecevic, M., Knezevic, M., Beyerlein, I.J., McCabe, R.J., 2016a. Origin of texture development in orthorhombic uranium. Materials Science and Engineering: A 665, 108-124.

Zecevic, M., Knezevic, M., Beyerlein, I.J., McCabe, R.J., 2016b. Texture formation in orthorhombic alpha-uranium under simple compression and rolling to high strains. Journal of Nuclear Materials 473, 143-156.

Zecevic, M., Knezevic, M., Beyerlein, I.J., Tomé, C.N., 2015a. An elasto-plastic self-consistent model with hardening based on dislocation density, twinning and de-twinning: Application to strain path changes in HCP metals. Materials Science and Engineering: A 638, 262-274.

Zecevic, M., McCabe, R.J., Knezevic, M., 2015b. A new implementation of the spectral crystal plasticity framework in implicit finite elements. Mechanics of Materials 84, 114-126.

Zecevic, M., McCabe, R.J., Knezevic, M., 2015c. Spectral database solutions to elastoviscoplasticity within finite elements: Application to a cobalt-based FCC superalloy. International Journal of Plasticity 70, 151-165. 